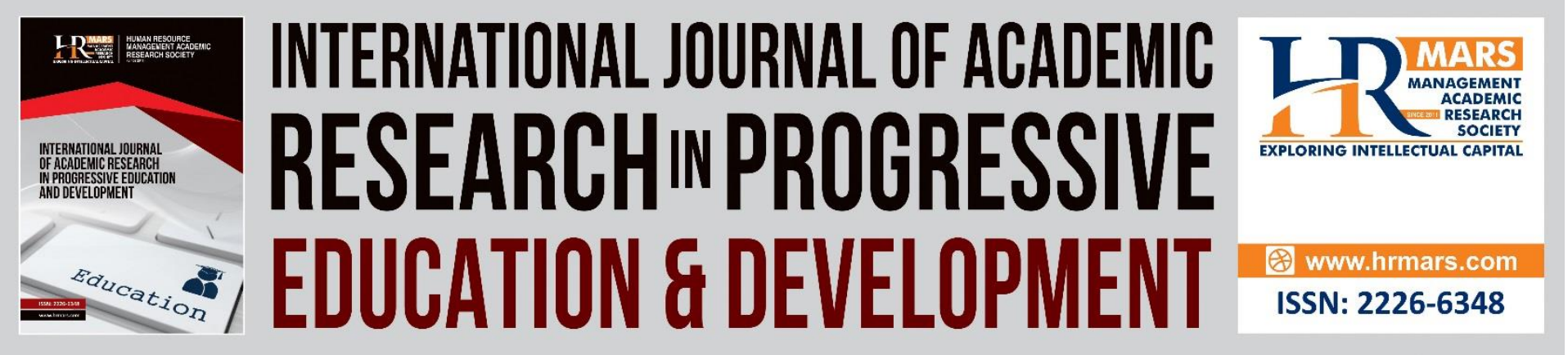

\title{
Translanguaging Pedagogy in the ESL Classroom: A Systematic Review
}

Wei Zhuang Ooi, Azlina Abdul Aziz

To Link this Article: http://dx.doi.org/10.6007/IJARPED/v10-i3/10816

DOI:10.6007/IJARPED/v10-i3/10816

Received: 07 June 2021, Revised: 15 July 2021, Accepted: 29 July 2021

Published Online: 26 August 2021

In-Text Citation: (Ooi \& Aziz, 2021)

To Cite this Article: Ooi, W. Z., \& Aziz, A. A. (2021). Translanguaging Pedagogy in the ESL Classroom: A Systematic Review. International Journal of Academic Research in Progressive Education and Development, 10(3), 676-709.

Copyright: (C) 2021 The Author(s)

Published by Human Resource Management Academic Research Society (www.hrmars.com)

This article is published under the Creative Commons Attribution (CC BY 4.0) license. Anyone may reproduce, distribute, translate and create derivative works of this article (for both commercial and non-commercial purposes), subject to full attribution to the original publication and authors. The full terms of this license may be seen at: http://creativecommons.org/licences/by/4.0/legalcode

Vol. 10(3) 2021, Pg. $676-709$

Full Terms \& Conditions of access and use can be found at http://hrmars.com/index.php/pages/detail/publication-ethics 


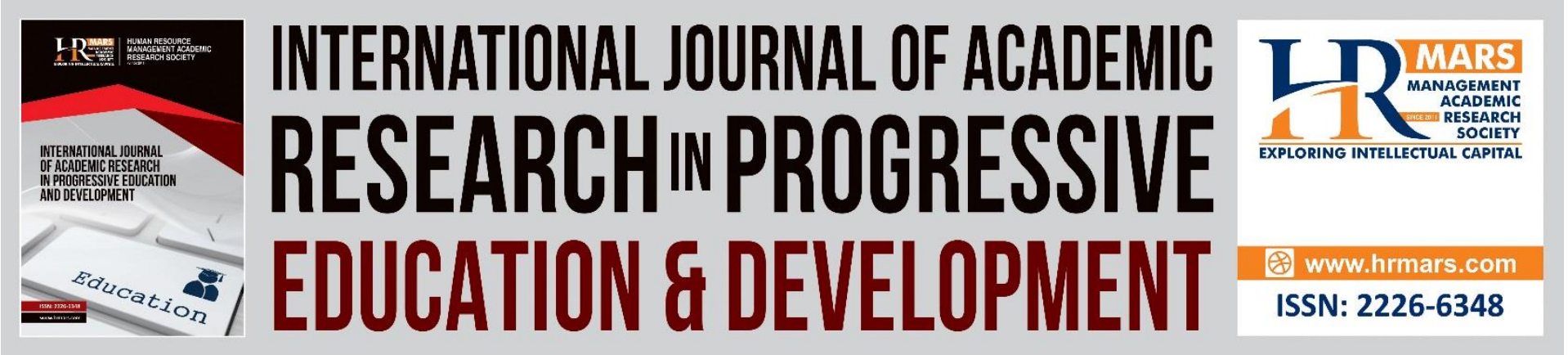

\title{
Translanguaging Pedagogy in the ESL Classroom: A Systematic Review
}

\author{
Wei Zhuang Ooi, Azlina Abdul Aziz \\ Faculty of Education, Universiti Kebangsaan Malaysia, 43000 UKM Bangi, Selangor, Malaysia \\ Email:weizhuangooi93@gmail.com, azlina1@ukm.edu.my
}

\begin{abstract}
The effects of technological advancements, globalization and social progress has enabled researchers and teachers to rediscover and innovate methods of English Language Teaching (ELT) to English as a Second Language (ESL) learners worldwide where more non-native speakers of English who identified as bilinguals or multilinguals enroll in English language classes to develop essential skills for international communication. Yet, teachers in ESL education stand by standardized and classic ELT methodology to teach all types of learners with strong emphasis on English-only use in the classroom in hopes of producing competent users of the English language. This long standing point of view has continually ignored bilingual and multilingual learners' potentials of learning a target language (L2) more effectively by accessing all linguistic resources from their first language (L1) or other familiar languages as a unified system through the practice of translanguaging. Therefore, this systematic literature review paper intends to explore the extent of current researches on translanguaging as an ESL pedagogy or ELT then highlight the potentials, considerations and trends of teaching and learning ESL with translingualism. A total 14 empirical journal articles related to the teaching practice were analysed to find out patterns in relevant research aims or purposes, research settings, research participants' education and multilingual backgrounds, research designs and data analysis used and considerations for ESL translanguaging pedagogy. This paper serves as a guide for ESL educators and researchers on the current use of translanguaging for developing ESL skills productively as well as recommendations for future research.
\end{abstract}

Keywords: Translanguaging, ESL, English as a Second Language, ELT, English Language Teaching, L1, L2

\section{Introduction}

English has become a necessary language to enable communication amongst people across the globe for transactional and interactional activities including scholarly pursuits, leisure, personal or professional development and establishing international relations to name a few. In fact, the ability to listen, speak, read and write in English is deemed essential to a degree that an estimation of $\mathbf{3 7 5}$ million English as a Second Language (ESL) learners are identified as bilinguals or multilinguals - individuals who speak two or more languages (Beare, 2020; 
Lüpke, 2020). As a result, there is a rise in bilinguals and multilinguals alike attending lessons to learn English in educational institutions worldwide. In contrast, teachers have continually practiced standard and classic English language teaching (ELT) methodology in the ESL classroom to teach the target language by immersing learners in an English-only environment with minimal interference or use of their first language (L1) and in turn have them acquire adequate language skills (Matamoros-González et al., 2017). This stance of advocating the use of purely English in ESL education while placing learners' use of first language as second-rate has been long standing with practitioners and students skeptical of the effectiveness of utilizing L1 when learning English.

However, with the advancement of technologies, globalization and social progress, English as a global language has made way for researchers and teachers to discover and innovate methods of ELT for multilinguals as the English learning classroom continues to include learners from diverse speech communities. Translanguaging has become an emergent strategy to develop bilingual or multilingual learners' ESL skills through simultaneous and systematic use of an individual's first language and English in teaching and learning sessions (Conteh, 2018; Lewis et al., 2012). As a matter of fact, translingualism has pedagogic potential in the education of ESL as demonstrated in various research (e.g., Akbar \& Taqi, 2020; Deroo \& Ponzio, 2019; Hu, 2020; Ting \& Jintang, 2020). Learners were allowed to use their existing linguistic knowledge in both L1 and target language (L2) to process and apply new language content through translanguaging between Malay and English in a Malaysian preschool (Ting \& Jintang, 2020). Besides, a discourse analysis among in-service teachers in the United States by Deroo and Ponzio (2019) suggested that translanguaging provide opportunities for collaboration between students and teachers to make sense of English learning, leverage learners' L1 as a learning resource for ESL and embrace learners' cultural background in the classroom. Akbar and Taqi also determined translanguaging as an ESL learning strategy works to effectively develop learners' language skills and their ability to express deeper levels of thinking through L2 in oral and written English exercises with accordance to their year 2020 case study in Kuwait. Last but not least, a review by $\mathrm{Hu}$ (2020) about existing studies of translanguaging in ESL classes concluded the practice benefits ESL pedagogy by efficiently delivering classroom instructions, clarifying linguistic content and enabling a sympathetic learning environment.

Yet, translanguaging is still a niche concept in the teaching and learning of ESL (Deroo \& Ponzio, 2019) which can possibly be mistaken for code-switching at times where it usually considers the switching of languages in discourse to be grammar focused and occurs as a result of using two separate languages where each language serves a separate function while one of the language use would appear dominant (Ferguson, 1959; Goodman \& Tastanbek, 2021; Kamwangamalu, 2010). In contrast, translanguaging is a bilingual's or multilingual's means of achieving learning and communicative goals by accessing their linguistic resources from various languages as a unified system (García, 2009). Hence, in spite of educators' adopting translanguaging in ELT for ESL, there is a deficit of literature reviews on the current researches on translingualism pedagogy in the ESL classroom. This systematic review paper extends the knowledge of translanguaging in current ESL education, its challenges and benefits and a clear direction for future research through exploring the extent of the concept in ESL teaching and learning from existing empirical studies. This paper will serve as a guide for ESL educators and researchers on the uses of translanguaging pedagogy for ESL skills development. Hence, this systematic review paper will investigate the following questions:

1. What were the purposes or aims in recent ESL translanguaging pedagogy research? 
DEVELOPMENT

Vol. 10, No. 3, 2021, E-ISSN: 2226-6348 @ 2021 HRMARS

2. Where were the research settings in the recent translanguaging studies?

3. Who were the research participants involved in the research designs and what was the nature of data analysis in the studies?

4. What were the multilingual backgrounds of the participants involved?

5. What were the considerations regarding ESL translanguaging pedagogy found in the studies?

\section{Literature Review}

\section{Shifting from Code-switching to Translanguaging}

The concept of codeswitching was initially discovered in social settings in which it was a language practice observed outside of the classroom among communities that speak more than one language in the $20^{\text {th }}$ century (Goodman \& Tastanbek, 2021). Also known as diglossia, code-switching ideologies view multilingualism or bilingualism as the practice of languages in separation where language systems do not overlap and are used based on different purposes designated by the individual or community (Ferguson, 1959). In addition to that, codeswitching holds a monoglossic view about languages to which monolingualism is a social norm and there is an unequal distinction between named languages used by multilinguals to pinpoint language dominance, elitism or proficiency in comparison (Gardner-Chloros, 2009; Myers-Scotton, 1993). With this in mind, code-switching was eventually integrated into the research of language learning but researchers felt limited by its fundamentals since codeswitching observed in general the interchange of languages in and between sentences with attention to linguistic structures (Del Valle, 2000; Lewis et al., 2012). Creese and Blackledge (2010) state that code-switching in the classroom was not established adequately for pedagogy purpose and was often referred to as a type of learning interference that disregards individuals' linguistic background and considers L1 use to be hindering language learning.

Thereupon, translanguaging was introduced by Williams $(1994,2000)$ through research on bilingualism among Welsh-English schools in Wales. Translanguaging looks beyond the delimiting principles in code-switching by taking into account all languages used in the teaching and learning of another language as heteroglossic and interrelated to allow effective target language development (García, 2009). Notably, Goodman and Tastanbek (2021) proposed a comprehensive framework for researchers and educators to transition from code-switching to translanguaging by highlighting the similarities and differences of each concept as seen in Figure 1. According to the framework, code-switching and translanguaging feature blending of known languages when multilinguals converse inside or outside of the classroom context. They can either occur unplanned or with intentions to strategically teach learning content. Teachers' and students' attitudes and application of both concepts are common around the world that goes against monolingual conventions and hegemony of English, believed to contribute negative or positive implications which can bring about changes in the class or social context. As compared to the mentioned distinct characteristics of codeswitching also found in the framework (e.g., Creese \& Blackledge, 2010; Del Valle, 2000; Ferguson, 1959; Gardner-Chloros, 2009; Lewis et al., 2012), translingualism is heteroglossic and views all signs or forms attempting to convey meaning as interconnected languages (Bailey, 2007; García, 2009). All languages are valued to benefit multilinguals' learning in many ways as they attempt to process language experiences logically or subjectively while developing a sound identity by harnessing their linguistic repertoire and resources at hand (García, 2009; MacSwan, 2017). As such, this study would be looking into translanguaging practices for purpose of capturing the dynamic and progressive disposition 


\section{DEVELOPMENT}

Vol. 10, No. 3, 2021, E-ISSN: 2226-6348 @ 2021 HRMARS

of language mixing, alternations, fusion and hybridity by multilinguals in education as supported by Garcia \& Wei (2014) and Goodman \& Tastanbek (2021).

Figure 1

Framework of the conceptual shift from code-switching to translanguaging

Both Codeswitching and Translanguaging

- Alternation, mixing, and hybridity/fusion of named languages in and out of class

- Language practices as spontaneous and/or intentionally pedagogical

- Teacher and student beliefs and practices as hegemonic, resistant, or transformative

Codeswitching

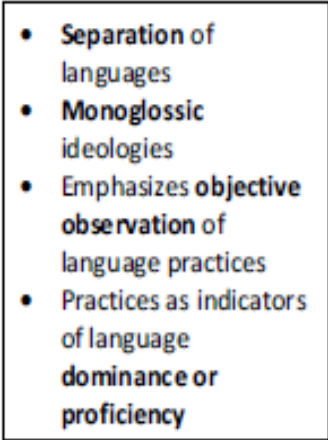

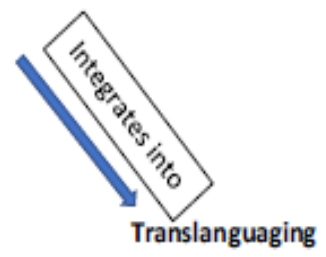

- Holistic view of languages

- Heteroglossic ideologies

- Emphasizes subjective understandings of language experiences

- Practices as paths to meaning making and identity formation

Note. This framework was produced by Goodman and Tastanbek in 2021, a conceptualization of similarities extending along code-switching and translanguaging, their differences and change towards translanguaging. From "Making the shift from a codeswitching to a translanguaging lens in English language teacher education," by B. Goodman and S. Tastanbek, 2021, TESOL Quarterly, 55(1), 29-53. Copyright 2021 by TESOL International Association.

\section{Translanguaging in English Language Teaching}

Language teaching for ESL in the past had not referred directly to translanguaging for its pedagogic potential although some traditional ELT methodology would support the use of L1 when teaching learners L2. These methodologies include Grammar Translation Method, Communicative Language Teaching and Natural Approach which stated incorporating L1 in teaching helps to assess, prompt interactions, bolster comprehension for language learning though some methodologies discouraged teachers to speak in L1 as they progress in learning or use L1 solely for the purpose of translation (Matamoros-González et al., 2017). MatamorosGonzález et al. (2017) went on to explain Audiolingualism which prohibits teachers and learners to use students' native language for the reason of language immersion along with efforts to develop students' English speaking better. 
Translanguaging can be seen as an emerging trend in the teaching of English amid language classrooms consisting greater numbers of multilinguals in recent times. Kleyn and García (2019) proposed translanguaging practices to be adopted in Teaching of English as a Second Language (TESL), Teaching of English to Speakers of Other Languages (ESOL) or teaching of English as a New Language (ENL) in response of reforming English language teaching and learning that were monolingual based in addition to acknowledge bilinguals' and multilinguals' unique practices of learning by employing full range of their linguistic resourcefulness. In particular, translanguaging practices for ESL are observable and feasible in classrooms when learners are allowed to learn or produce English with flexible use of other languages when making sense of the target language content. Some instances are identified in Ofelia García's and Hesson's (2015) translanguaging framework for educators in which bilingual or multilingual students can access multilingual materials, ask questions or express themselves with their language repertoire before, during and after reading, writing, speaking and listening English language tasks. As for teachers, they can provide multilingual instructions or feedback, create a safe place for multilingual interactions, model translanguaging, allow students to explain themselves with their language resources, assign peer support for translanguaging as means of ensuring that students can understand then relate to the content of four English language skills.

In Malaysia, a study by Ting and Jintang (2020) analysed interactions of 15 preschoolers amongst those who speak Malay, Chinese, Bidayuh and Iban natively from two classrooms with two teachers. Relatively, translanguaging between English and Malay language during an English lesson developed skills of students for both languages as they learn new English vocabulary and concepts by encouraging pre-school children to use languages they know resourcefully. Teacher-student translanguaging talks were found to be significant for communicative, management and academic operations in the classroom. Translanguaging practices prompt responses from preschoolers positively and comfortably which is crucial in expanding their cognitive abilities.

In another research, Deroo and Ponzio (2019) conducted a discourse analysis with inservice teachers regarding their views on translanguaging in the United States. Data collected and analysed from five teachers over six weeks revealed teachers who were bilinguals and English monolinguals to be supportive of translanguaging practices and multilingualism. It was suggested that exposure to translingualism theories or practices dispel biased monolingual ideologies of teaching as the preferable method or norm for ESL education. Translanguaging principles adopted by teachers are essential for acknowledging and appreciating bilinguals' or multilinguals' full capabilities in learning through consolidating learners' L1 literacy along with teachers' ability to communicate in learners' L1 as well. The enactment of translanguaging in ESL education will need endorsement from educators, administrators, communities and policy makers alike.

Likewise, Akbar and Taqi (2020) investigated effects of translanguaging on 34 bilingual college students' language performance and learning. Result from students' English writing skill assessment indicated improved scores especially in aspects of inferencing for information and explaining complex ideas with slight improvements on language proficiency and basic information comprehension. In other words, translanguaging may be more effective for developing learners' higher thinking skills next to their language proficiency. Nevertheless, the study also showed learners who practice translingualism were able to cultivate confidence and reduce their anxiety when learning in the ESL classroom that subsequently activated their ability to process information using all of their language resources. 
DEVELOPMENT

Vol. 10, No. 3, 2021, E-ISSN: 2226-6348 @ 2021 HRMARS

Based on an article titled "Translanguaging in ESL/EFL Classes: A Review Paper" (Hu, 2020), the author reviewed past papers related to translanguaging ranging from the year 2009-2019. Of the seven studies analysed, it can be concluded that translanguaging was generally used for three purposes: classroom control, explain linguistic content and establish positive learning environment. Rationale of translanguaging refrain of use were observed to be based on strict monolingual government language policies and fear of learners' poor development of L2 proficiency. Some suggestions were provided for further studies such as proving of language skills improvement using quantitative tests and extensive or longer research terms on the effects of translanguaging in ESL education.

Given these points of discussion, this systematic literature review paper seeks to bridge the gap and further extend information on current researches of translanguaging for the teaching and learning of ESL and relevant pedagogy concepts from state-of-the-art empirical research articles.

\section{Methodology}

This study used a systematic review method to inspect existing literature, identify and appraise the contributions followed by the analysis, synthesis and reporting of data based on the method's detailed and planned approach that can avoid bias selection commonly found in traditional literature reviews (Briner \& Denyer, 2012; Uman, 2011). The purpose of this review is to inform ESL educators and researchers on the recent studies about translanguaging pedagogy in ESL education. Correspondingly, the researchers of this study conducted a systematic review observing the five steps proposed by Khan et al. (2003) which were: 1) framing questions for a review 2) identifying relevant work 3 ) assessing the quality of studies 4) summarizing the evidence and lastly, 5) interpreting the findings. The reviewed literature pieces were located in Google Scholar database whereas the literature search procedure was conducted during June 2021. This systematic review enabled methodical organization of existing research findings as evidence to inform best practices, trends and gaps for decision making (Hart, 2018).

\section{Step 1: Framing Questions for a Review}

Technology has evolved education by providing modern tools to discover useful or relevant knowledge and skills about languages. This is evident in ESL education as the practice of translanguaging has gained traction in transforming ESL pedagogy. This study aims to analyse recent empirical studies on the extent of translanguaging research done and its pedagogy in the ESL learning context, published from 2017 to 2021. The research questions include: i) What were the purposes or aims in recent ESL translanguaging pedagogy research? ii) Where were the research settings in the recent translanguaging studies? iii) Who were the research participants involved in the research designs and what was the nature of data analysis in the studies? iv) What were the multilingual backgrounds of the participants involved? v) What were the considerations regarding ESL translanguaging pedagogy found in the studies?

\section{Step 2: Identifying Relevant Work}

Google Scholar was accessed as the main database to collect relevant existing literature as data for this study. First, the terms inputted into Google Scholar were "translanguaging", "esl", "english as a second language", "L1", "L2" "ELT", "English language teaching", pedagogy, bilingual, multilingual and -book. Relevant articles were collected and then further refined for analysis from researchers' inclusion and exclusion criteria. In addition, 
literature distributed through unconventional means, known as grey literature, including dissertations, policy documents, proceedings and conference abstracts that may reflect questionable quality were omitted in this study to minimize bias (Hopewell et al., 2005). Thereupon, the articles collected were limited to peer-reviewed full-text articles published in journals to ensure methodical quality of the studies found (Hopewell et al., 2005). Referring to the purpose of this systematic review, empirical studies were chosen from 28 full-text articles found in Google Scholar. This was because that empirical data from these studies report provide valuable first-hand experiences (Neuman, 2014) to comprehensively explain practices of translanguaging in ESL education. Notably, this study focused on published empirical studies dated between 2017-2021, within the recent five years to depict latest research on translingualism in the ESL education field.

\section{Step 3: Assessing the Quality of Studies}

An inclusion and exclusion criteria listed elements to inspect the qualities of articles collected and further pinpoint suitable articles applicable to this research. Table 1 shows the inclusion and exclusion criteria set for this review.

\section{Table 1}

Inclusion and exclusion criteria

\begin{tabular}{|c|c|}
\hline Inclusion criteria & Exclusion criteria \\
\hline $\begin{array}{l}\text { Empirical studies examining } \\
\text { translanguaging pedagogy in ESL education }\end{array}$ & $\begin{array}{l}\text { Studies examining translanguaging outside } \\
\text { of ESL education }\end{array}$ \\
\hline $\begin{array}{l}\text { Empirical studies partially investigating } \\
\text { translanguaging pedagogy in ESL education }\end{array}$ & $\begin{array}{l}\text { Studies that investigate translanguaging } \\
\text { pedagogy but do not facilitate process of } \\
\text { ESL acquisition }\end{array}$ \\
\hline $\begin{array}{l}\text { Articles distributed in peer-reviewed } \\
\text { journals }\end{array}$ & $\begin{array}{l}\text { Articles not distributed in peer-reviewed } \\
\text { journals }\end{array}$ \\
\hline $\begin{array}{l}\text { Peer-reviewed journal articles with results } \\
\text { and discussions }\end{array}$ & $\begin{array}{l}\text { Journal articles without results and } \\
\text { discussions }\end{array}$ \\
\hline $\begin{array}{l}\text { Journal articles published between the } \\
\text { years } 2017 \text { and } 2021\end{array}$ & $\begin{array}{l}\text { Journal articles not published between the } \\
\text { years } 2017 \text { and } 2021\end{array}$ \\
\hline \multirow[t]{2}{*}{ Full-text peer-reviewed journal articles } & Articles in grey literature \\
\hline & Articles with access to abstract only \\
\hline
\end{tabular}

\section{Step 4: Summarizing the Evidence}

The literature search process was completed in June 2021 with the search terms keyed into selected databases: "translanguaging", "esl", "english as a second language", "L1", "L2" "ELT", "English language teaching", pedagogy, bilingual, multilingual and -book. There was a total of 82 results found from Google Scholar. A total of 81 results remained after duplicates were removed. Then, the results were screened and 53 results were excluded. 28 full-text articles 
were collected and read through which led to 14 full-text articles being excluded. Finally, 14 peer-reviewed empirical journal articles were selected for analysis in this systematic review. The literature search process is outlined in a PRISMA flow chart adapted from Moher et al. (2009) in Figure 2.

\section{Step 5: Interpreting the Findings}

Content analysis was applied to interpret the systematic review findings and answer the formulated research questions. A total of 14 full-text empirical journal articles about translanguaging used in teaching and learning of ESL were analysed. Table 2 exhibits an analysis of the research methodology used in the published empirical studies from 2017 to 2021. Based on the table, mixed method studies were most prevalent when researching the use of online interactive learning tools for ESL education, followed by qualitative and quantitative studies. Participant involved in all the relevant studies ranged from primary, secondary, tertiary levels of ESL education. 


\section{Figure 2}

\section{PRISMA Flow Chart}
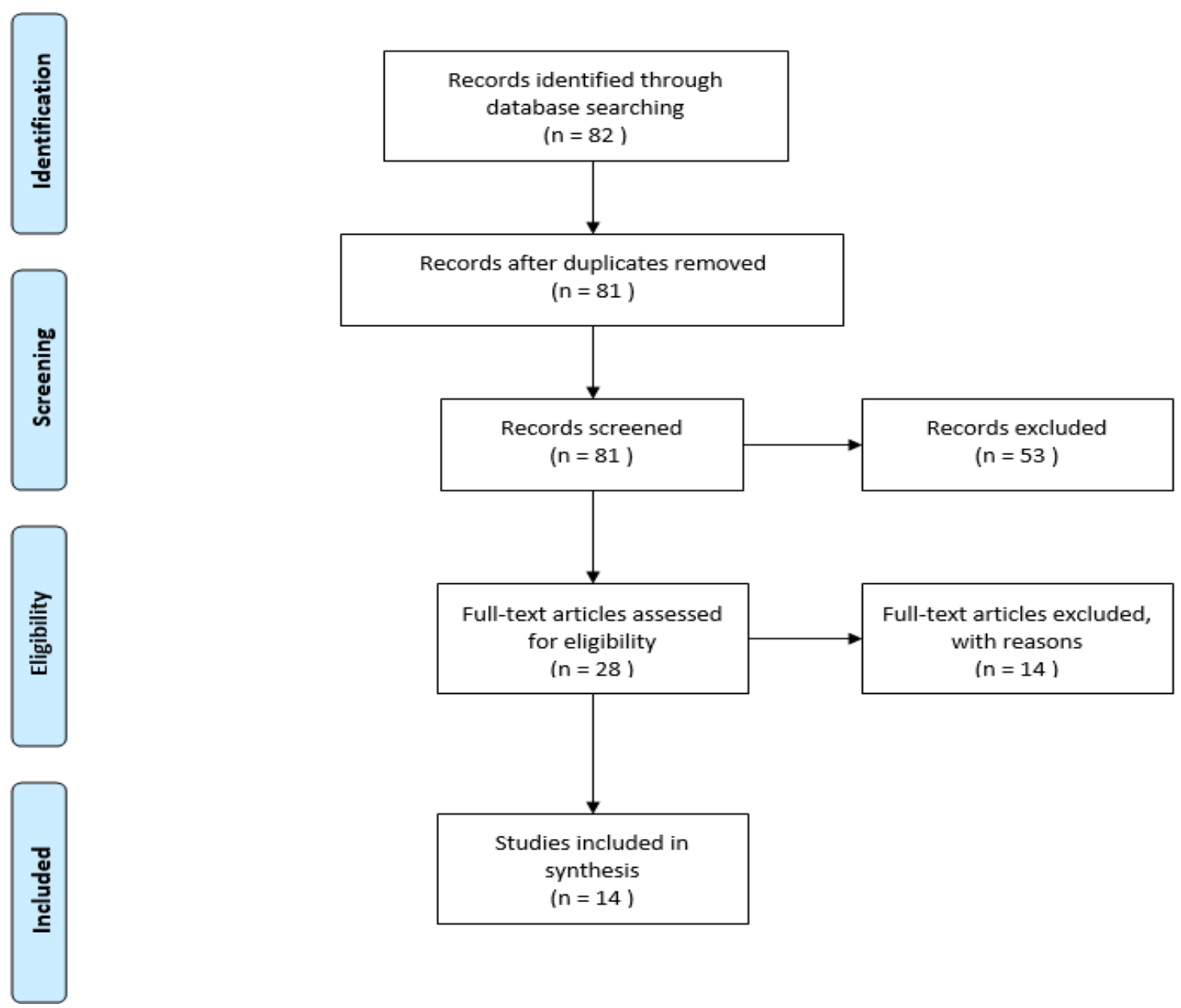

Note. This flow chart was adapted and used for the systematic literature review process of this paper. Adapted from "Preferred Reporting Items for Systematic Reviews and Meta-Analyses: The PRISMA Statement," by D. Moher, A. Liberati, J. Tetzlaff, D. G. Altman and The PRISMA Group, 2009, PLOS Med, 6(7), e100009. (https://doi.org/10.1371/journal.pmed.1000097). Copyright by PLOS.

\section{Table 2}

Numbers of Study Based on Research Methodology

\begin{tabular}{|l|l|}
\hline $\begin{array}{l}\text { Research Methodology of Empirical } \\
\text { Articles }\end{array}$ & $\begin{array}{l}\text { Number Empirical Research Articles from Google } \\
\text { Scholar }\end{array}$ \\
\hline Quantitative & 2 \\
\hline Qualitative & 6 \\
\hline Mixed Method & 6 \\
\hline Total Articles & 14 \\
\hline
\end{tabular}


INTERNATIONAL JOURNAL OF ACADEMIC RESEARCH IN PROGRESSIVE EDUCATION AND

DEVELOPMENT

Vol. 10, No. 3, 2021, E-ISSN: 2226-6348 @ 2021 HRMARS

\section{Table 3}

Literature Synthesis Matrix of existing studies related to translanguaging in ESL education and pedagogy

\begin{tabular}{|c|c|c|c|c|c|c|c|}
\hline \multicolumn{2}{|c|}{$\begin{array}{l}\text { Information } \\
\text { of Article - } \\
\text { Author(s) } \\
\text { (Year) }\end{array}$} & $\begin{array}{l}\text { Purpose } \\
\text { or aims of } \\
\text { research }\end{array}$ & $\begin{array}{l}\text { Research } \\
\text { setting }\end{array}$ & $\begin{array}{l}\text { Research } \\
\text { participants' } \\
\text { education } \\
\text { background }\end{array}$ & $\begin{array}{l}\text { Multilingua } \\
\text { I } \\
\text { background } \\
\mathrm{s} \\
\text { research of } \\
\text { participants }\end{array}$ & $\begin{array}{l}\text { Research } \\
\text { design and } \\
\text { nature of } \\
\text { data } \\
\text { analysis } \\
\end{array}$ & $\begin{array}{l}\text { Considerations } \\
\text { regarding } \\
\text { translanguaging } \\
\text { pedagogy }\end{array}$ \\
\hline 1 & $\begin{array}{l}\text { Wong } \\
\& \\
\text { Yoong } \\
(2019)\end{array}$ & $\begin{array}{l}\text { find out } \\
\text { ESL } \\
\text { teachers' } \\
\text { views } \\
\text { towards } \\
\text { code- } \\
\text { switching } \\
\text { in } \\
\text { classroom; } \\
\text { how does } \\
\text { code- } \\
\text { switching } \\
\text { happen in } \\
\text { the ESL } \\
\text { classroom }\end{array}$ & $\begin{array}{l}\text { Malaysia } \\
\text { Chinese } \\
\text { Independe } \\
\mathrm{nt} \\
\text { Secondary } \\
\text { School }\end{array}$ & $\begin{array}{l}7 \text { ESL teachers, } \\
\text { tertiary level } \\
\text { education } \\
\text { graduates }\end{array}$ & $\begin{array}{l}\text { Chinese, } \\
\text { English, } \\
\text { Malay }\end{array}$ & $\begin{array}{l}\text { Naturalistic } \\
\text { approach } \\
\text { Qualitative } \\
\text { data } \\
\text { analysis }\end{array}$ & $\begin{array}{l}\text { Translanguaging } \\
\text { - Facilitates learning } \\
\text { for grammar, lexical } \\
\text { items } \\
\text { - Allows assessment of } \\
\text { learning } \\
\text { - Increase learning } \\
\text { participation } \\
\text { - Build rapport and } \\
\text { connection with } \\
\text { learners } \\
\text { Convey opinions, } \\
\text { thoughts, ideas and } \\
\text { feelings of educators } \\
\text { to learners' learning } \\
\text { behaviour } \\
\text { Improve language } \\
\text { learning among } \\
\text { lower language } \\
\text { proficiency and } \\
\text { lesser motivated } \\
\text { learns } \\
\text { Continuous use of } \\
\text { target language may } \\
\text { cause learning } \\
\text { anxiety and negative } \\
\text { perception of target } \\
\text { language }\end{array}$ \\
\hline 2 & $\begin{array}{l}\text { Eisenst } \\
\text { ein } \\
\text { Ebswor } \\
\text { th et al. } \\
\text { (2018) }\end{array}$ & $\begin{array}{l}\text { investigat } \\
\text { e } \\
\text { perceptio } \\
\text { ns, } \\
\text { experienc } \\
\text { es and } \\
\text { suggestion } \\
\mathrm{s} \text { about } \\
\text { English }\end{array}$ & $\begin{array}{l}\text { education } \\
\text { al } \\
\text { institution } \\
\text { s of Puerto } \\
\text { Rico }\end{array}$ & $\begin{array}{l}86 \text { experienced } \\
\text { English } \\
\text { teachers in } \\
\text { Puerto Rico } \\
\text { with } \\
\text { elementary, } \\
\text { secondary, } \\
\text { college or a } \\
\text { combination of }\end{array}$ & $\begin{array}{l}\text { Spanish, } \\
\text { English }\end{array}$ & $\begin{array}{l}\text { Constructivi } \\
\text { st and } \\
\text { classical } \\
\text { grounded } \\
\text { theory } \\
\text { approach } \\
\text { Qualitative } \\
\text { and }\end{array}$ & $\begin{array}{l}\text { - Learners should be } \\
\text { allowed to use all } \\
\text { their linguistic } \\
\text { resources to relate } \\
\text { and make sense of } \\
\text { target language } \\
\text { learnt } \\
\text { Bilingualism } \\
\text { biliteracy: }\end{array}$ \\
\hline
\end{tabular}


INTERNATIONAL JOURNAL OF ACADEMIC RESEARCH IN PROGRESSIVE EDUCATION AND

DEVELOPMENT

Vol. 10, No. 3, 2021, E-ISSN: 2226-6348 @ 2021 HRMARS

\begin{tabular}{|c|c|c|c|c|c|c|c|}
\hline & & $\begin{array}{l}\text { acquisitio } \\
\mathrm{n} \quad \text { from } \\
\text { English } \\
\text { teachers } \\
\text { in Puerto } \\
\text { Rico }\end{array}$ & & $\begin{array}{l}\text { two and more } \\
\text { levels of } \\
\text { learners }\end{array}$ & & $\begin{array}{l}\text { quantitative } \\
\text { data } \\
\text { analysis }\end{array}$ & $\begin{array}{l}\text { - Can be promoted to } \\
\text { retain native culture } \\
\text { and first language } \\
\text { - Can enrich learners' } \\
\text { professional and } \\
\text { personal } \\
\text { development while } \\
\text { respecting their } \\
\text { background with } \\
\text { meaningful content } \\
\text { relevant to one's } \\
\text { culture }\end{array}$ \\
\hline 3 & $\begin{array}{l}\text { Qiu \& } \\
\text { Fang } \\
(2019)\end{array}$ & $\begin{array}{l}\text { Investigat } \\
\text { e China } \\
\text { university } \\
\text { students } \\
\text { view of } \\
\text { local } \\
\text { teachers } \\
\text { and native } \\
\text { English } \\
\text { speakers } \\
\text { as EMI } \\
\text { teachers' } \\
\text { teaching } \\
\text { behaviour; } \\
\text { similarities } \\
\text { and } \\
\text { difference } \\
\text { s of } \\
\text { classroom } \\
\text { practices } \\
\text { among } \\
\text { two } \\
\text { groups of } \\
\text { EMI teachers } \\
\text { to }\end{array}$ & $\begin{array}{l}\text { University } \\
\text { in China }\end{array}$ & $\begin{array}{l}101 \text { university } \\
\text { students in } \\
\text { EMI } \\
\text { programmes } \\
\text { with at least six } \\
\text { years of } \\
\text { English } \\
\text { learning } \\
\text { experience, } \\
\text { aged } 18 \text { to } 23\end{array}$ & $\begin{array}{l}\text { Chinese, } \\
\text { English }\end{array}$ & $\begin{array}{l}\text { Quantitativ } \\
\text { e and } \\
\text { qualitative } \\
\text { research } \\
\text { design } \\
\text { Quantitativ } \\
\text { e and } \\
\text { qualitative } \\
\text { data } \\
\text { analysis }\end{array}$ & $\begin{array}{l}\text { - Translanguaging } \\
\text { practices using } \\
\text { multimodal devices } \\
\text { and L1 enhance } \\
\text { teaching efficiency } \\
\text { - English-only } \\
\text { classrooms can } \\
\text { cause } \\
\text { miscommunication } \\
\text { due to language } \\
\text { barrier } \\
\text { Educators should } \\
\text { know learner's L1 to } \\
\text { some extent } \\
\text { Educators who speak } \\
\text { learner's first } \\
\text { language tend have } \\
\text { comprehensible } \\
\text { English accents for } \\
\text { learners } \\
\text { Educators who are } \\
\text { native speakers of } \\
\text { learners' } \\
\text { language are more } \\
\text { familiar with their } \\
\text { learning and thinking } \\
\text { styles as well as } \\
\text { provide students } \\
\text { with purpose of } \\
\text { learning by relating } \\
\text { content knowledge } \\
\text { to international and } \\
\text { domestic } \\
\text { implications }\end{array}$ \\
\hline
\end{tabular}




\begin{tabular}{|c|c|c|c|c|c|c|c|}
\hline & & & & & & & $\begin{array}{l}\text { - Intracultural and } \\
\text { intercultural } \\
\text { competence can } \\
\text { improve } \\
\text { communication then } \\
\text { learning } \\
\text { effectiveness } \\
\end{array}$ \\
\hline 4 & $\begin{array}{l}\text { Amin et } \\
\text { al. } \\
(2020)\end{array}$ & $\begin{array}{l}\text { Explore } \\
\text { students' } \\
\text { view of } \\
\text { using } \\
\text { English, } \\
\text { Urdu and } \\
\text { other } \\
\text { regional } \\
\text { languages } \\
\text { in } \\
\text { education; } \\
\text { role of } \\
\text { English in } \\
\text { defining } \\
\text { the } \\
\text { significanc } \\
\text { e of their } \\
\text { L1 and } \\
\text { other } \\
\text { regional } \\
\text { languages; } \\
\text { reasons } \\
\text { students } \\
\text { prefer } \\
\text { English } \\
\text { over L1 } \\
\text { and other } \\
\text { languages } \\
\text { in education } \\
\text { eder }\end{array}$ & $\begin{array}{l}\text { Pakistani } \\
\text { Universitie } \\
\text { s }\end{array}$ & $\begin{array}{l}200 \text { students } \\
\text { from social } \\
\text { sciences, } 200 \\
\text { students from } \\
\text { natural } \\
\text { sciences in four } \\
\text { public sector } \\
\text { universities }\end{array}$ & $\begin{array}{l}\text { Urdu, other } \\
\text { Pakistani } \\
\text { regional } \\
\text { languages: } \\
\text { Pashto, } \\
\text { Punjabi, } \\
\text { Balochi, } \\
\text { Sindhi,, } \\
\text { English }\end{array}$ & $\begin{array}{l}\begin{array}{l}\text { Survey } \\
\text { research } \\
\text { design }\end{array} \\
\text { Quantitativ } \\
\text { e data } \\
\text { analysis }\end{array}$ & $\begin{array}{l}\text { - Heavy emphasis on } \\
\text { English language } \\
\text { education can } \\
\text { undermine the } \\
\text { importance of } \\
\text { learners' L1 and their } \\
\text { regional languages } \\
\text { - Use of English and } \\
\text { regional languages } \\
\text { should be } \\
\text { accommodated by } \\
\text { teachers and } \\
\text { students an } \\
\text { stakeholders may } \\
\text { view both languages } \\
\text { to be valuable assets } \\
\text { Use of regional } \\
\text { languages and target } \\
\text { language an } \\
\text { education an an } \\
\text { eliminate linguistic } \\
\text { disparities and } \\
\text { provide for learners' } \\
\text { linguistic needs and } \\
\text { preferences } \\
\text { Language policies } \\
\text { can encourage } \\
\text { translanguaging } \\
\text { practices a }\end{array}$ \\
\hline 5 & $\begin{array}{l}\text { Tan \& } \\
\text { Low } \\
(2017)\end{array}$ & $\begin{array}{l}\text { Find out } \\
\text { attitudes } \\
\text { of } \\
\text { teachers } \\
\text { towards } \\
\text { code- } \\
\text { switching } \\
\text { in ESL } \\
\text { classroom } \\
\mathrm{s} \text { of rural }\end{array}$ & $\begin{array}{l}\text { National } \\
\text { primary } \\
\text { schools } \\
\text { and } \\
\text { national- } \\
\text { type } \\
\text { Chinese } \\
\text { primary } \\
\text { schools in } \\
\text { a rural }\end{array}$ & $\begin{array}{l}62 \text { teachers } \\
\text { from three } \\
\text { large national } \\
\text { primary } \\
\text { schools, small } \\
\text { national } \\
\text { primary } \\
\text { schools, three } \\
\text { large national- } \\
\text { type (Chinese) }\end{array}$ & $\begin{array}{l}\text { Malay, } \\
\text { Chinese, } \\
\text { English }\end{array}$ & $\begin{array}{l}\text { Survey } \\
\text { research } \\
\text { design } \\
\text { Quantitativ } \\
\text { e data } \\
\text { analysis }\end{array}$ & $\begin{array}{l}\text { - ESL teachers } \\
\text { teaching in rural } \\
\text { areas may employ } \\
\text { translanguaging and } \\
\text { support additive } \\
\text { bilingualism view } \\
\text { - Translanguaging } \\
\text { uses an individual's } \\
\text { linguistic resource to }\end{array}$ \\
\hline
\end{tabular}


INTERNATIONAL JOURNAL OF ACADEMIC RESEARCH IN PROGRESSIVE EDUCATION AND

DEVELOPMENT

Vol. 10, No. 3, 2021, E-ISSN: 2226-6348 @ 2021 HRMARS

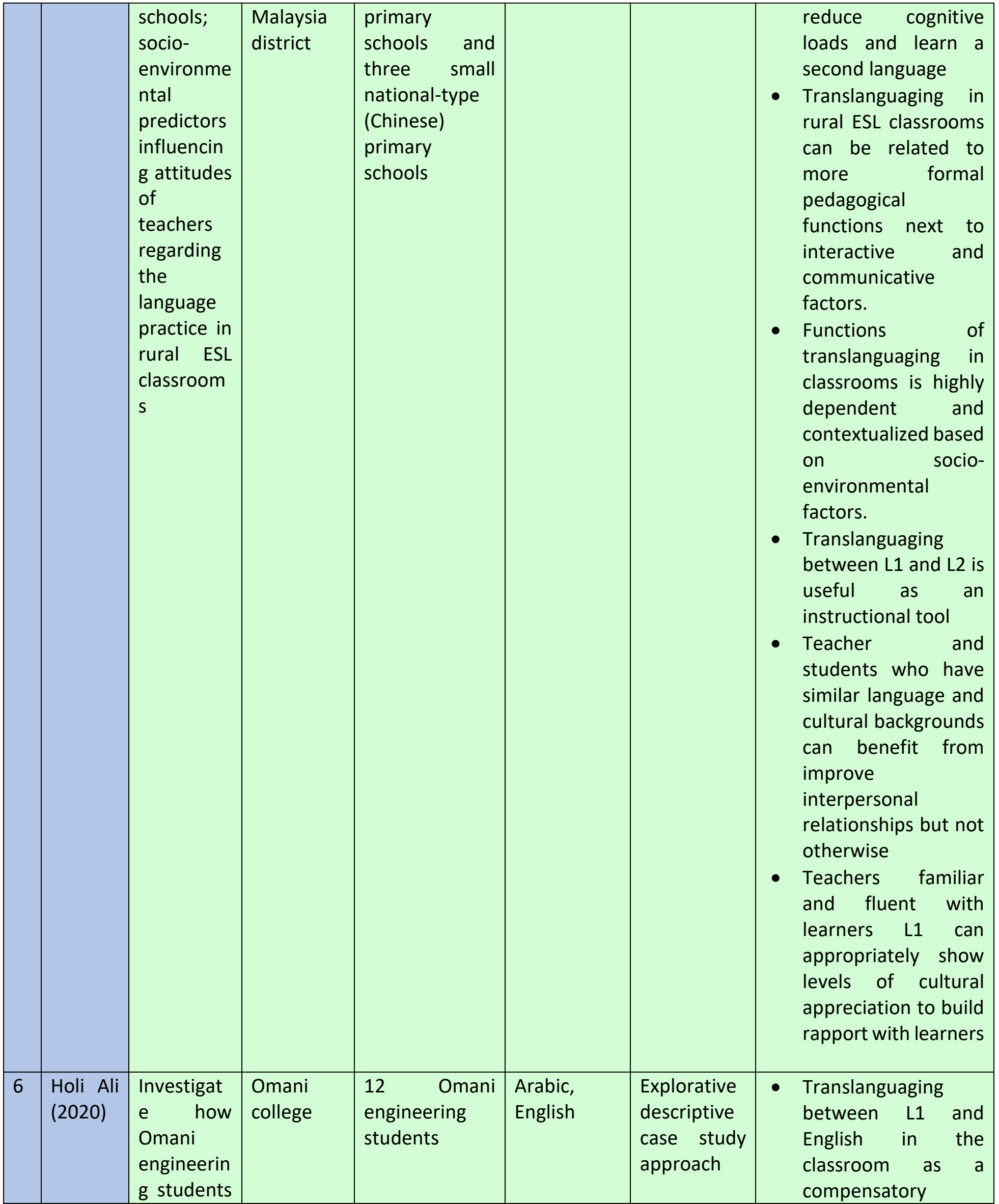


INTERNATIONAL JOURNAL OF ACADEMIC RESEARCH IN PROGRESSIVE EDUCATION AND

DEVELOPMENT

Vol. 10, No. 3, 2021, E-ISSN: 2226-6348 @ 2021 HRMARS

\begin{tabular}{|c|c|c|c|c|c|c|c|}
\hline & & \begin{tabular}{|l} 
used \\
coping \\
strategies \\
when \\
studying \\
English \\
Medium \\
Instructio \\
$n \quad$ (EMI) \\
courses
\end{tabular} & & & & $\begin{array}{l}\text { Qualitative } \\
\text { data } \\
\text { analysis }\end{array}$ & $\begin{array}{l}\text { strategy to reduce } \\
\text { miscommunications } \\
\text { and improve } \\
\text { understanding of } \\
\text { learning content } \\
\text { - Translanguaging can } \\
\text { improve learners' L1 } \\
\text { and L2 skills } \\
\text { altogether } \\
\text { - Translanguaging } \\
\text { with L1 may be } \\
\text { perceived by some } \\
\text { learners to be } \\
\text { counterintuitive } \\
\text { - Learners may also } \\
\text { need to be able to } \\
\text { use translanguaging } \\
\text { among peers to } \\
\text { scaffold learning } \\
\text { element of a } \\
\text { language } \\
\text { Translanguaging } \\
\text { with L1 can be } \\
\text { utilized clarification, for } \\
\text { emphasis, summary } \\
\text { and reiteration of } \\
\text { crucial learning } \\
\text { contents } \\
\text { It helps build rapport } \\
\text { between educators } \\
\text { and learners, } \\
\text { increase classroom } \\
\text { management efficiency and } \\
\text { alleviate disciplinary } \\
\text { issues }\end{array}$ \\
\hline 7 & $\begin{array}{l}\text { Im } \\
(2020)\end{array}$ & $\begin{array}{l}\text { Find out } \\
\text { how } \\
\text { Korean } \\
\text { speakers } \\
\text { of English } \\
\text { can be } \\
\text { accounted } \\
\text { in the } \\
\text { translingu }\end{array}$ & $\begin{array}{l}\text { Korean } \\
\text { media }\end{array}$ & 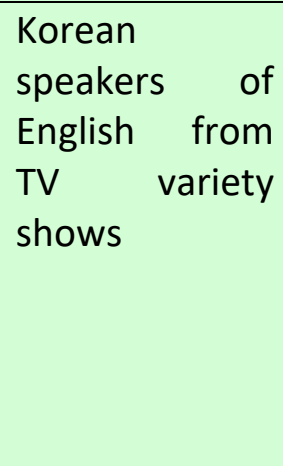 & $\begin{array}{l}\text { Korean, } \\
\text { English }\end{array}$ & \begin{tabular}{|l} 
Discourse \\
analysis \\
informed by \\
conversatio \\
$\mathrm{n}$ analysis
\end{tabular} & $\begin{array}{l}\text { - Translingualism } \\
\text { focuses on effective } \\
\text { communication and } \\
\text { lesser need on } \\
\text { languages used } \\
\text { being grammatically } \\
\text { accurate } \\
\text { - ESL teachers should } \\
\text { consider learners to }\end{array}$ \\
\hline
\end{tabular}




\begin{tabular}{|c|c|c|c|c|c|c|c|}
\hline & & \begin{tabular}{|l} 
alism \\
approach; \\
their \\
translingu \\
al \\
strategies; \\
lessons \\
learnt \\
from \\
translingu \\
alism by \\
Korean \\
teachers \\
of English
\end{tabular} & & & & $\begin{array}{l}\text { Qualitative } \\
\text { data } \\
\text { analysis }\end{array}$ & $\begin{array}{l}\text { use forms of English } \\
\text { they know as with } \\
\text { the occurrence of } \\
\text { translingualism to } \\
\text { negotiate meaning } \\
\text { and interact with } \\
\text { others } \\
\text { - Learners should be } \\
\text { allowed to employ } \\
\text { linguistic, cultural } \\
\text { and contextual } \\
\text { resources of L1 and } \\
\text { L2 when encoding } \\
\text { and decoding } \\
\text { meaning during } \\
\text { interactions } \\
\text { - Translingualism } \\
\text { allow learners to } \\
\text { learn English with } \\
\text { the goal of English } \\
\text { communication } \\
\text { competence in mind } \\
\text { as they mutually } \\
\text { construct meaning } \\
\text { with other English } \\
\text { speakers using } \\
\text { various interactional } \\
\text { strategies }\end{array}$ \\
\hline 8 & $\begin{array}{l}\text { Schreib } \\
\text { er } \\
\text { (2019) }\end{array}$ & \begin{tabular}{|l} 
Investigat \\
e \\
outcomes \\
of online \\
intercultur \\
al \\
exchange \\
among MA \\
TESL \\
students \\
with New \\
York \\
undergrad \\
uate \\
students; \\
how \\
interactio \\
ns with \\
diverse
\end{tabular} & $\begin{array}{l}\text { Sri Lankan } \\
\text { university, } \\
\text { New York } \\
\text { college }\end{array}$ & $\begin{array}{l}12 \text { student } \\
\text { groups from } \\
\text { Master of Arts } \\
\text { TESL program } \\
\text { Sri Lankan } \\
\text { University } \\
\text { Postgraduate } \\
\text { Students and } \\
\text { New York } \\
\text { college } \\
\text { undergraduate } \\
\text { students }\end{array}$ & \begin{tabular}{|l} 
Sri Lankan \\
students: \\
Sinhala, \\
Tamil, \\
English \\
\\
New York \\
students: \\
Chinese \\
Spanish, \\
Russian, \\
Arabic, \\
Gujarati, \\
Burmese, \\
Serbo- \\
Croatian, \\
Tagalog, \\
Haitian \\
Creole,
\end{tabular} & $\begin{array}{l}\text { Grounded } \\
\text { content } \\
\text { analysis } \\
\text { approach } \\
\text { Qualitative } \\
\text { data } \\
\text { analysis }\end{array}$ & 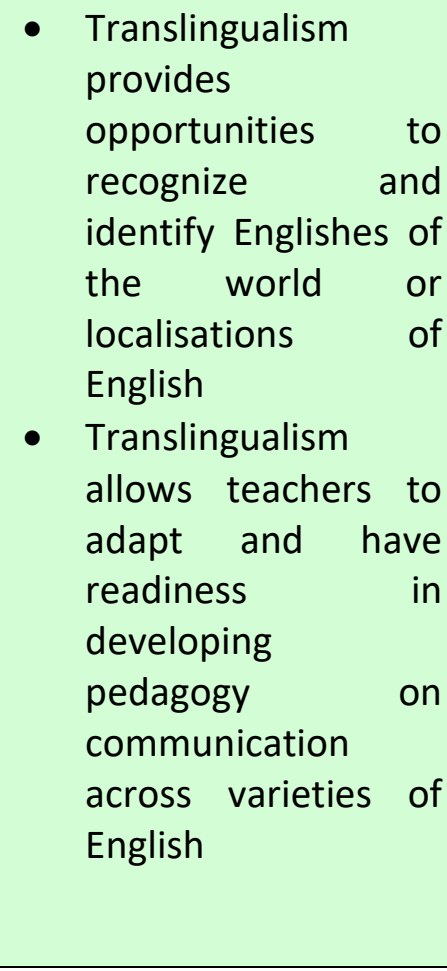 \\
\hline
\end{tabular}


DEVELOPMENT

Vol. 10, No. 3, 2021, E-ISSN: 2226-6348 ㄷ 2021 HRMARS

\begin{tabular}{|c|c|c|c|c|c|c|c|}
\hline & & \begin{tabular}{|l} 
English \\
speaking \\
groups \\
impact Sri \\
Lankan \\
students' \\
perceptio \\
ns of \\
English \\
native \\
speakers \\
as \\
compared \\
to \\
themselve \\
$\mathrm{s} \quad$ as L2 \\
speakers.
\end{tabular} & & & \begin{tabular}{|l|} 
African \\
American \\
Vernacular \\
English, \\
dialects of \\
New York \\
English
\end{tabular} & & 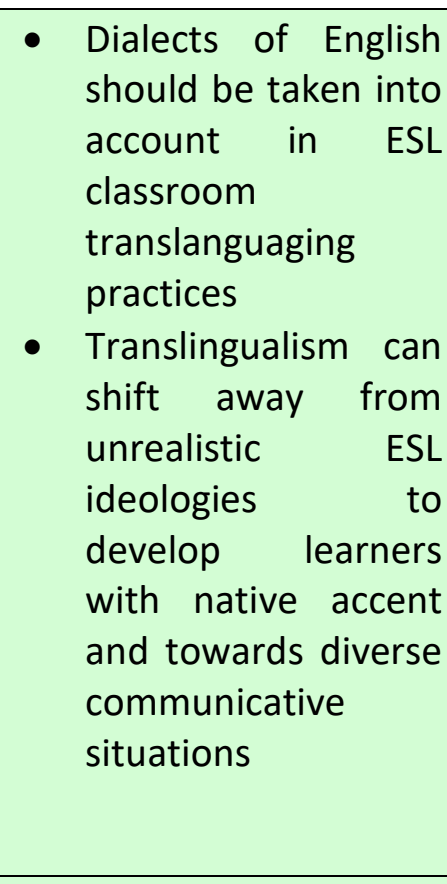 \\
\hline 9 & $\begin{array}{l}\text { Lindahl } \\
\text { (2020) }\end{array}$ & $\begin{array}{l}\text { Explore } \\
\text { extent of } \\
\text { content } \\
\text { and } \\
\text { language } \\
\text { integrated } \\
\text { learning } \\
\text { (CLIL) } \\
\text { educators } \\
\text { use Critical } \\
\text { Multilingu } \\
\text { al } \\
\text { Language } \\
\text { Awarenes } \\
\text { s (CMLA) } \\
\text { to analyse } \\
\text { student } \\
\text { utterances } \\
; \\
\text { extent the } \\
\text { language } \\
\text { ideologies } \\
\text { emerging } \\
\text { in student } \\
\text { utterances }\end{array}$ & $\begin{array}{l}\text { Western } \\
\text { USA public } \\
\text { school } \\
\text { district }\end{array}$ & $\begin{array}{l}53 \text { educators } \\
\text { working with } \\
\text { elementary } \\
\text { and middle } \\
\text { schoolers in a } \\
\text { bilingual } \\
\text { format } \\
\text { through CLIL } \\
\text { programme }\end{array}$ & $\begin{array}{l}\text { French, } \\
\text { Spanish, } \\
\text { English }\end{array}$ & $\begin{array}{l}\begin{array}{l}\text { Qualitative } \\
\text { survey } \\
\text { research }\end{array} \\
\text { Qualitative } \\
\text { data } \\
\text { analysis }\end{array}$ & $\begin{array}{l}\text { - ESL educators who } \\
\text { believe in } \\
\text { standardization of } \\
\text { English and } \\
\text { practicing the } \\
\text { language with solely } \\
\text { one language may } \\
\text { hinder learners' } \\
\text { process of } \\
\text { understanding target } \\
\text { language through } \\
\text { translanguaging } \\
\text { practices } \\
\text { - Translanguaging } \\
\text { pedagogy should be } \\
\text { considered to allow } \\
\text { educators } \\
\text { understand reasons } \\
\text { and ways learners' } \\
\text { use or acquire a } \\
\text { language }\end{array}$ \\
\hline $\begin{array}{l}1 \\
0\end{array}$ & $\begin{array}{l}\text { Tsang \& } \\
\text { Lo } \\
\text { (2020) }\end{array}$ & $\begin{array}{l}\text { Find out } \\
\text { effects of } \\
\text { pluri- } \\
\text { centric } \\
\text { designed }\end{array}$ & $\begin{array}{l}\text { language- } \\
\text { enrichmen } \\
\text { t course in } \\
\text { Hong Kong }\end{array}$ & $\begin{array}{l}\text { 14 primary } \\
\text { school children } \\
\text { in Hong Kong }\end{array}$ & $\begin{array}{l}\text { Cantonese, } \\
\text { English }\end{array}$ & \begin{tabular}{|l} 
Mixed- \\
method \\
case study
\end{tabular} & $\begin{array}{ll}\text { - } & \text { Translanguaging } \\
\text { practices } & \text { in } \\
\text { multilingual } & \text { ESL } \\
\text { classroom } & \text { can }\end{array}$ \\
\hline
\end{tabular}




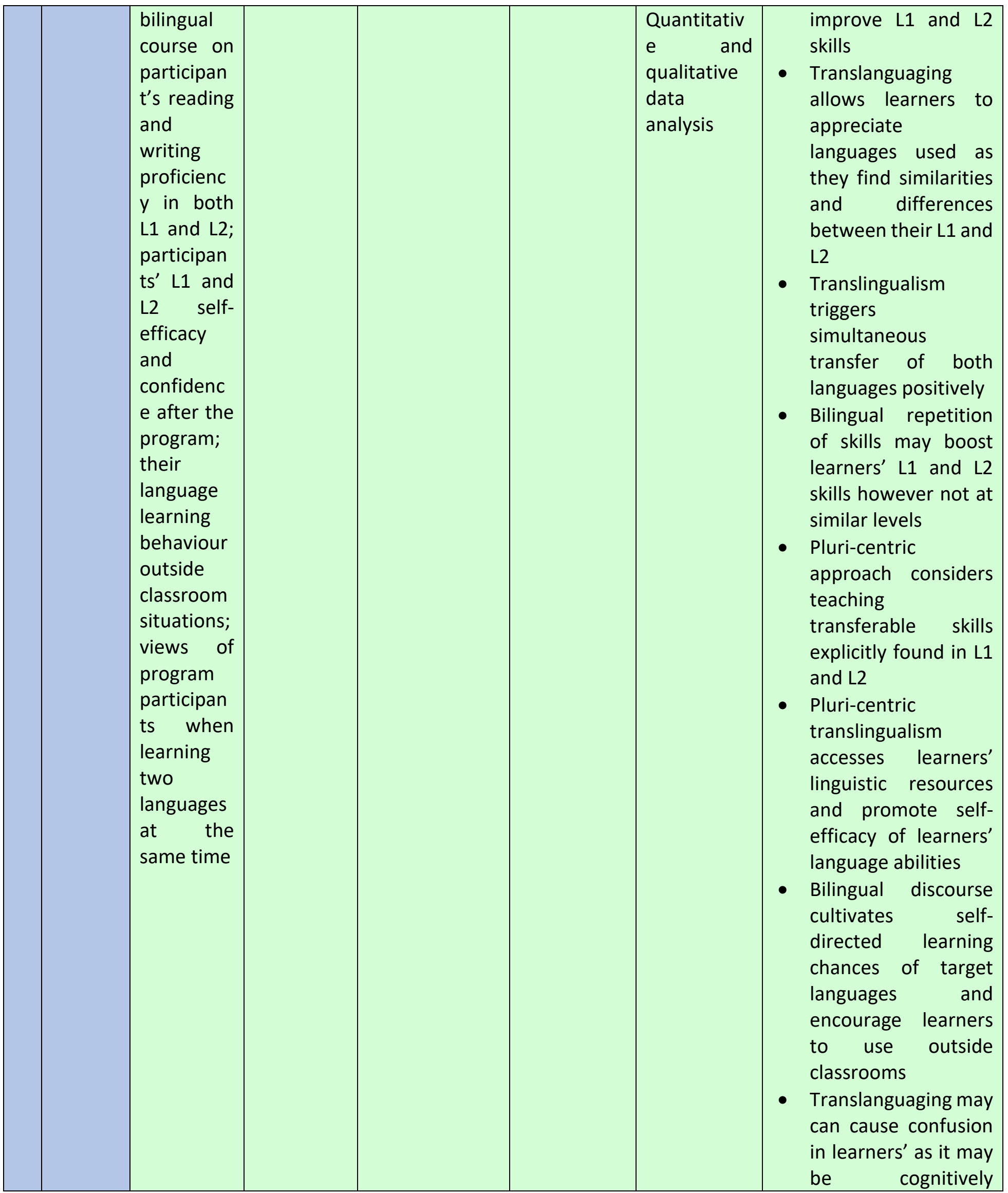




\begin{tabular}{|c|c|c|c|c|c|c|c|}
\hline & & & & & & & $\begin{array}{l}\text { demanding for some } \\
\text { if not motivated }\end{array}$ \\
\hline 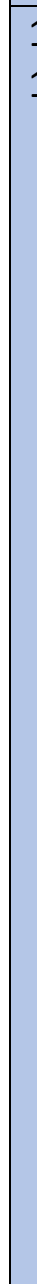 & $\begin{array}{l}\text { Macaro } \\
\text { et al. } \\
(2020)\end{array}$ & $\begin{array}{l}\text { Find out } \\
\text { the ratio } \\
\text { of L1 and } \\
\text { L2 in } \\
\text { teacher } \\
\text { talk in } \\
\text { English } \\
\text { Medium } \\
\text { Instructio } \\
n \quad \text { (EMI) } \\
\text { courses; } \\
\text { teachers' } \\
\text { purpose of } \\
\text { L1 use; } \\
\text { students' } \\
\text { thoughts } \\
\text { of using } \\
\text { both } \\
\text { languages } \\
\text { in EMI } \\
\text { classroom } \\
\mathrm{s}\end{array}$ & $\begin{array}{l}\text { University } \\
\text { in China }\end{array}$ & $\begin{array}{l}\text { Five EMI } \\
\text { teachers and } \\
165 \text { students } \\
\text { partaking in } \\
\text { EMI courses in } \\
\text { a Beijing } \\
\text { University }\end{array}$ & $\begin{array}{l}\text { Chinese, } \\
\text { English }\end{array}$ & 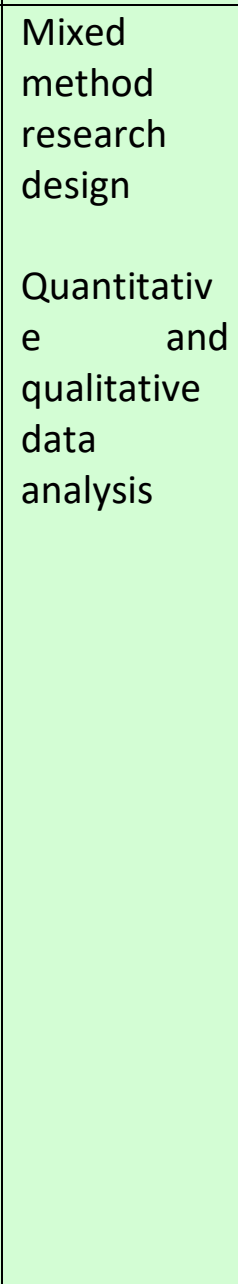 & $\begin{array}{l}\text { - Translanguaging } \\
\text { practices are for } \\
\text { explaining target } \\
\text { language, academic } \\
\text { content that is } \\
\text { conceptually } \\
\text { complex or demand } \\
\text { higher cognitive } \\
\text { load. } \\
\text { - Translingualism can } \\
\text { activate learners' } \\
\text { prior knowledge to } \\
\text { relate new } \\
\text { information } \\
\text { meaningfully } \\
\text { - Translanguaging } \\
\text { occur lesser with } \\
\text { learners that are } \\
\text { more proficient in L2 } \\
\text { and may resort to L1 } \\
\text { to } \\
\text { communication solve } \\
\text { breakdowns or } \\
\text { misunderstandings if } \\
\text { required only }\end{array}$ \\
\hline $\begin{array}{l}1 \\
2\end{array}$ & $\begin{array}{l}\text { Lindahl } \\
\text { et al. } \\
(2020)\end{array}$ & $\begin{array}{l}\text { Determine } \\
\text { how } \\
\text { teachers' } \\
\text { Critical } \\
\text { Multilingu } \\
\text { al } \\
\text { Language } \\
\text { Awarenes } \\
\text { s (CMLA) is } \\
\text { affected } \\
\text { by their } \\
\text { experienc } \\
\text { es of } \\
\text { studying } \\
\text { abroad; } \\
\text { how CMLA } \\
\text { principles } \\
\text { guide and } \\
\text { impact }\end{array}$ & $\begin{array}{l}\text { Universitie } \\
\text { s in Costa } \\
\text { Rica, } \\
\text { Dominican } \\
\text { Republic, } \\
\text { Mexico }\end{array}$ & $\begin{array}{l}27 \text { North Texan } \\
\text { pre-service } \\
\text { teacher, } 20 \text { in- } \\
\text { service } \\
\text { teachers in } \\
\text { New York } \\
\text { pursuing } \\
\text { TESOL } \\
\text { Certifications, } \\
9 \text { South Texan } \\
\text { pre-service ESL } \\
\text { and bilingual } \\
\text { teachers }\end{array}$ & $\begin{array}{l}\text { Spanish, } \\
\text { English }\end{array}$ & $\begin{array}{l}\text { Qualitative } \\
\text { survey } \\
\text { research } \\
\\
\text { Qualitative } \\
\text { data } \\
\text { analysis }\end{array}$ & 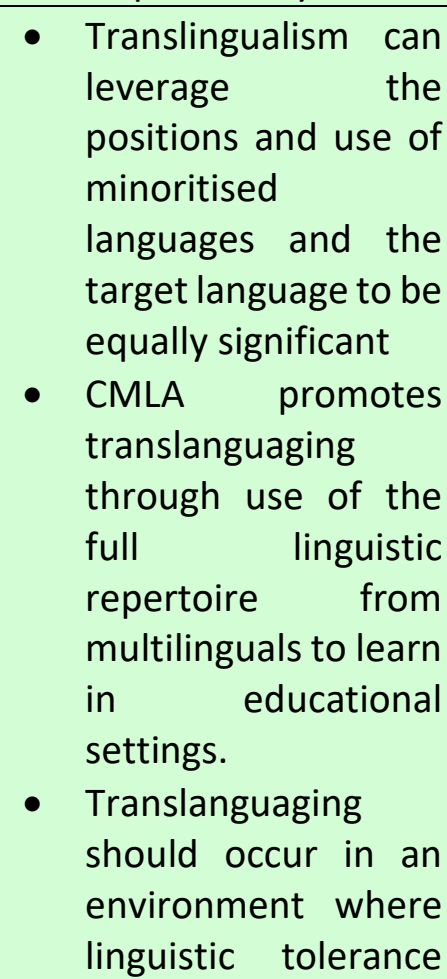 \\
\hline
\end{tabular}


DEVELOPMENT

Vol. 10 , No. 3, 2021, E-ISSN: 2226-6348 @ 2021 HRMARS

\begin{tabular}{|c|c|c|c|c|c|c|c|}
\hline & & \begin{tabular}{|l} 
teachers \\
to \\
understan \\
$d \quad$ study \\
abroad \\
program \\
designs
\end{tabular} & & & & & $\begin{array}{l}\text { and plurilingualism } \\
\text { awareness is present } \\
\text { among educators } \\
\text { and learners }\end{array}$ \\
\hline 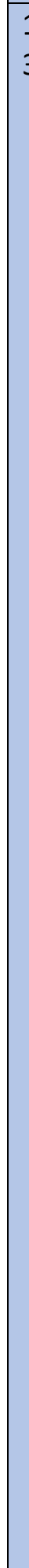 & $\begin{array}{l}\text { Turnbul } \\
\text { I (2017) }\end{array}$ & $\begin{array}{l}\text { Investigat } \\
\text { e views of } \\
\text { future ESL } \\
\text { teachers } \\
\text { about } \\
\text { using L1 } \\
\text { during L2 } \\
\text { classroom } \\
\text { s; reasons } \\
\text { for the } \\
\text { pre- } \\
\text { service } \\
\text { teachers' } \\
\text { views; } \\
\text { future } \\
\text { implicatio } \\
\text { ns for use } \\
\text { of native } \\
\text { languages } \\
\text { in L2 } \\
\text { classroom } \\
\text { in ESL } \\
\text { teacher } \\
\text { training } \\
\text { courses }\end{array}$ & $\begin{array}{l}\text { University } \\
\text { in New } \\
\text { Zealand }\end{array}$ & $\begin{array}{l}30 \quad \mathrm{TESOL} \\
\text { undergraduate } \\
\text { students }\end{array}$ & $\begin{array}{l}\text { English, } \\
\text { Korean, } \\
\text { Chinese, } \\
\text { Vietnamese }\end{array}$ & $\begin{array}{l}\text { Quantitativ } \\
\text { e and } \\
\text { qualitative } \\
\text { survey } \\
\text { research } \\
\text { Quantitativ } \\
\text { e and } \\
\text { qualitative } \\
\text { data analysis } \\
\text { analis }\end{array}$ & 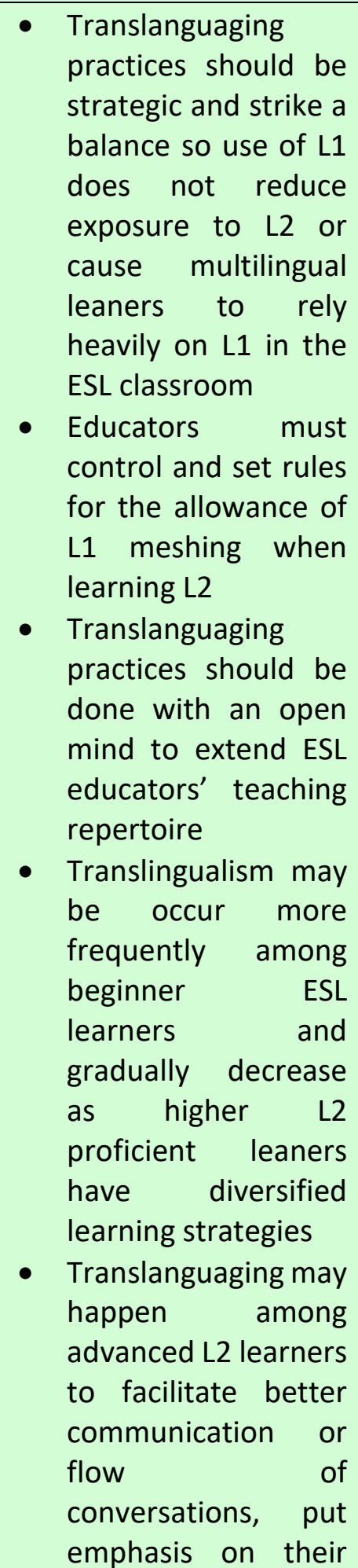 \\
\hline
\end{tabular}




\begin{tabular}{|c|c|c|c|c|c|c|c|}
\hline & & & & & & & $\begin{array}{l}\text { messages conveyed, } \\
\text { compensate or learn } \\
\text { L2 vocabulary or } \\
\text { grammar and aid in } \\
\text { advanced problem- } \\
\text { solving. }\end{array}$ \\
\hline 1 & $\begin{array}{l}\text { Akbar } \\
\& \text { Taqi } \\
\text { (2020) }\end{array}$ & $\begin{array}{l}\text { Investigat } \\
\text { e if } \\
\text { translangu } \\
\text { aging } \\
\text { enhances } \\
\text { bilingual } \\
\text { students' } \\
\text { academic } \\
\text { performan } \\
\text { ce; the } \\
\text { effectiven } \\
\text { ess of } \\
\text { translangu } \\
\text { aging } \\
\text { developin } \\
\text { g learners' } \\
\text { ESL; } \\
\text { learners' } \\
\text { attitude of } \\
\text { translangu } \\
\text { aging as an } \\
\text { ESL } \\
\text { teaching } \\
\text { method }\end{array}$ & $\begin{array}{l}\text { College in } \\
\text { Kuwait }\end{array}$ & $\begin{array}{l}34 \text { female } \\
\text { bilingual } \\
\text { students from } \\
\text { a } \\
\text { psycholinguisti } \\
\mathrm{c} \text { program } \\
\text { belonging to } \\
\text { the English } \\
\text { Department in } \\
\text { college }\end{array}$ & $\begin{array}{l}\text { Arabic, } \\
\text { English }\end{array}$ & 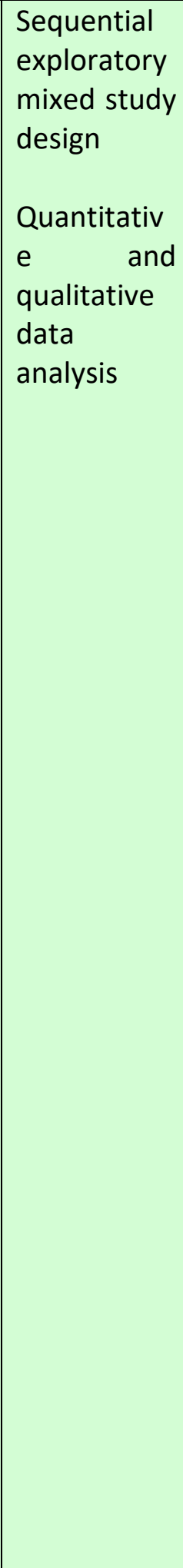 & 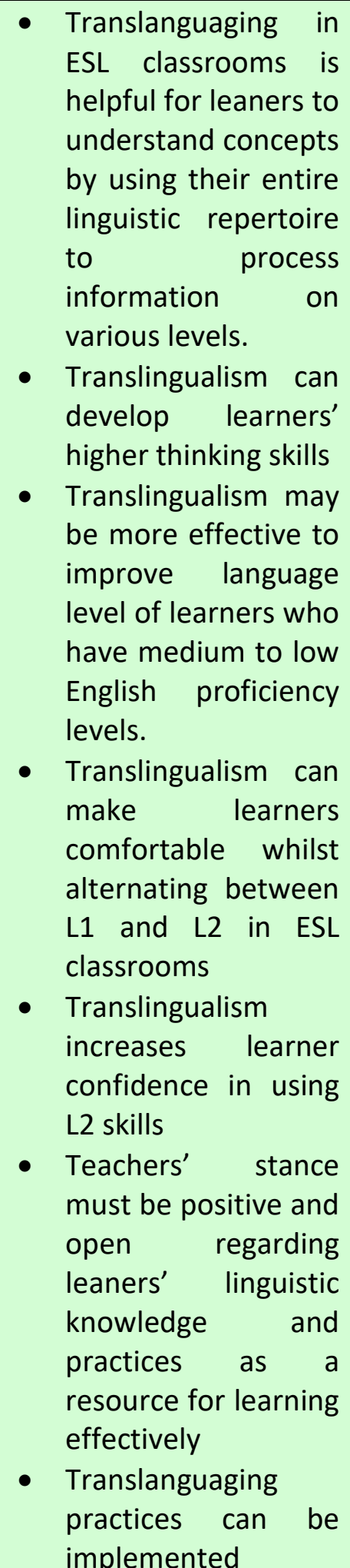 \\
\hline
\end{tabular}


INTERNATIONAL JOURNAL OF ACADEMIC RESEARCH IN PROGRESSIVE EDUCATION AND

DEVELOPMENT

Vol. 10, No. 3, 2021, E-ISSN: 2226-6348 ㄷ 2021 HRMARS

\begin{tabular}{|l|l|l|l|l|l|}
\hline & & & & & \\
\end{tabular}




\section{Findings and Discussion}

The research findings were tabulated and synthesised according to the analysis of 14 journal articles identified from the research criteria in Table 3. Relevant data was extracted and interpreted in order to answer the 5 research questions. All of the selected empirical journal articles had discussed aims or purposes of its research, the research setting, education background of the research participants, participants' multilingual background, the research design and nature of data analysis of its research and considerations of translanguaging ESL pedagogy. From the findings, discussions were carried out following the research questions devised.

\section{RQ 1: What were the purposes or aims in recent ESL translanguaging pedagogy research?}

Based on the 14 journal articles, most articles have similar aims or purposes when researching related subject matters to translanguaging pedagogy in the ESL classroom. Eight articles attempted to investigate directly the practice of $\mathrm{L} 1$ in English language learning classes, codeswitching or translanguaging between $\mathrm{L} 1$ and English as the target language (e.g., Akbar \& Taqi, 2020; Amin et al., 2020; Im, 2020; Macaro et al., 2020; Tan \& Low, 2017; Tsang \& Lo, 2020; Turnbull, 2017; Wong \& Yoong, 2019). Specifically, seven of the eight articles inquired their research participants about attitudes, perception and views regarding utilization of L1 or other languages when teaching and learning about ESL in the classroom with Akbar and Taqi, Amin et al., Macaro et al. and Tsang and Lo gathering information from students' perspective in the year 2020 while Wong and Yoong (2019); Tan and Low (2017), and Turnbull (2017) focused on teachers' or pre-service teachers' perspective of translanguaging elements in the ESL classroom. Factors influencing teachers' views were scrutinized as a follow up in Tan's and Low's (2017); Turnbull's (2017) research in addition of implications for training ESL teachers in the latter.

Next, four studies examined the reasons for the occurrence of translanguaging practices. Macaro et al. (2020) along with Wong and Yoong (2019) had research purposes of finding out how teachers' use or functions of L1 in ESL lessons where students' reasons of L1 usage were explained by Amin et al. (2020). Asides from that, Im (2020) researched how Korean speakers of English in general use translanguaging approach and strategies situationally and the implications derived for teachers of the English language in Korea educational institutions.

The effects of translanguaging on learners were assessed in two research articles. Students' $L 1$ and $L 2$ reading and writing proficiency together with self-efficacy about the two languages and learning behaviours regarding L1 and L2 outside the classroom were inspected after Tsang and Lo (2020) had students completed an educational program that simultaneously taught L1 and L2 skills. Another study by Akbar and Taqi (2020) tested learners' learning performance after utilizing translanguaging as a language learning strategies in the ESL classroom.

A total of six articles reviewed had research purposes that did not reference translanguaging directly but discussed concepts of translingualism with English as the L2 as an outcome in their research (e.g., Eisenstein Ebsworth et al., 2018; Ali, 2020; Lindahl, 2020; Lindahl et al., 2020; Qiu \& Fang, 2019; Schreiber, 2019). Holi Ali (2020) as well as Qiu and Fang (2019) carried out their studies in English Medium Instruction (EMI) university courses where students attend classes about a subject matter in English in order to learn content in addition to L2. The former found out strategies used by Omani students to cope learning in 
EMI courses and the latter was about Chinese university students' perceptions of local and native English-speaking teachers' teaching practices in EMI courses with comparison of the two teacher groups' classroom practices. On the other hand, Critical Multilingual Language Awareness (CMLA) of teachers and pre-service teachers were analysed in studies by Lindahl (2020) and Lindahl et al. (2020). Both studies analysed teachers' knowledge, sensitivity and appreciation towards learners' ability use of more than one languages with the motive of consolidating all languages learners acquired or are learning at the moment. The first explored Content and Language Integrated Learning (CLIL) teachers practice of CMLA in relation to student talk in the classroom. The second research with aims related to CMLA determined the effects of studying abroad on teachers' language awareness and to what degree CMLA principles guided teachers when participating in study abroad programmes. As for Eisenstein Ebsworth et al. (2018), the authors studied on the perspectives and experiences of English teachers in Puerto Rico on how learners acquire the English language then provided suggestions for the teaching and learning of English as the target language. Lastly, a research by Schreiber (2019) inspected the effects of intercultural exchange among Sri Lankan postgraduate master degree students and undergraduate students from New York. The impacts of the programme were also analysed in conjunction to Sri Lankan students' selfperception as L2 speakers.

In general, the purposes and aims of recent research of translanguaging pedagogy for ESL regarded mostly on the views of teachers and students about translanguaging followed by even distribution on the investigations of L1 roles in translanguaging, influencing factors on teachers' or students' perception of translanguaging and effects of translanguaging in the English language learning classroom. Translanguaging pedagogy research were also found in studies relating to ESL acquisition, CMLA of teachers and EMI university courses where use of L1 was deliberated when non-native English speakers learn in English. Therefore, latest research on translanguaging pedagogy have substantial data on students' and teachers' perspective of the practice.

\section{RQ 2: Where were the research settings in the recent translanguaging studies?}

In terms of the research setting of the 14 articles reviewed, 12 different settings were identified. Among the articles, two research were conducted in Malaysian rural district national and national-type Chinese primary schools and in a Chinese independent secondary school respectively by Tan and Low (2017) and Wong and Yoong (2019). Two other articles from Macaro et al. (2020) and Qiu \& Fang (2019) carried out their research in different universities in China. The ten remaining research settings of the remainder reviewed articles (e.g., Akbar \& Taqi, 2020; Amin et al., 2020; Eisenstein Ebsworth et al., 2018; Holi Ali, 2020; Im, 2020; Lindahl, 2020; Lindahl et al., 2020; Schreiber, 2019; Tsang \& Lo, 2020; Turnbull, 2017) were from a college in Kuwait; universities in Pakistan; educational institutions in Puerto Rico; an Omani college; the Korean media; the western United States of America public school district; universities in Costa Rica, Dominican Republic and Mexico; a Sri Lankan university and a New York college; a language-enrichment course in Hong Kong; and, a university in New Zealand according to the articles cited in order. Hence, recent research about translanguaging pedagogy was not particularly concentrated in a geographical setting but all research revolved around the theme of translanguaging in the ESL teaching and learning context. 


\section{RQ 3: Who were the research participants involved in the research designs and what was the nature of data analysis in the studies?}

As for the research participants involved in the 14 recent research articles relating to translanguaging ESL pedagogy, four categories were identified according to their education backgrounds and occupation. The research designs and nature of data analysis established in the studies were also analysed in this section in conjunction to the research participants of each study. To point out, six studies had research participants who were teachers and preservice teachers, five studies with undergraduate or postgraduate students, one study included both teachers and undergraduate students, another involving primary school children and lastly one study with people found in media as research participants. Wong and Yoong (2019) used the naturalistic approach as a qualitative research design in order to collect and analyse data qualitatively from seven ESL teachers with tertiary level education qualifications teaching in a Malaysian Chinese Independent Secondary School. A group of 53 elementary and secondary CLIL programme educators provided qualitative data for analysis through a qualitative survey research design deployed by Lindahl (2020). Similarly, 27 preservice North Texan teachers, 20 in-service New York teachers in the process of gaining Teaching English to Speakers of Other Languages certification and 9 pre-service South Texan English language teachers conducted a qualitative survey research with Lindahl et al. (2020). Data from the survey research were analysed qualitatively. In contrast, a survey research design was adopted and quantitative data analysis was carried out among 62 Malaysian teachers from six national primary schools together with 6 national-type Chinese primary schools by Tan and Low (2017). Next, 88 experienced English teachers working in elementary, secondary, college level education institutions of Puerto Rico were involved in Eisenstein Ebsworth's et al. (2018) research. The authors adopted the constructivist and classical grounded theory approach for their research and analysed data gathered from English teachers of Puerto Rico both qualitatively and quantitatively. A different research article by Turnbull (2017) had 30 pre-service ESL teachers joined a quantitative and qualitative survey research. The data collected from the author's survey analysed quantitatively and qualitatively suitable for discussions of translanguaging ESL pedagogy.

In relation to research articles with undergraduate students, 101 China university students aged 18 to 23 with minimum six years of English learning experience enrolled in EMI programmes participated in a quantitative and qualitative research by Qiu and Fang (2019) while the data was analysed accordingly. Schreiber (2019) gathered data from 12 groups of students consisting of Sri Lankan university Masters of Arts TESL postgraduates and New York college undergraduates. A grounded content analysis approach and qualitative data analysis was done for the study. Likewise, a sequential exploratory mixed method research design was adopted in Akbar and Taqi's (2020) study with analysis of data done quantitatively and qualitatively. A total of 34 female bilingual students undertaking a psycholinguistic course from the English department of a Kuwait college participated in the authors' research. A survey research design with quantitative analysis of data among 200 social sciences students and 200 natural sciences students from four public Pakistani universities was completed by Amin et al (2020). Oppositely, a study linked to translanguaging ESL pedagogy used an explorative descriptive case study approach to analyse qualitative data from 12 Omani engineering college students enrolled in EMI courses (Ali, 2020).

The remainder three studies reviewed in this research were from Macaro et al (2020), Tsang and Lo (2020) and Im (2020) where their research designs were based on a mixed 
method, a mixed-method case study and a discourse analysis informed by conversation analysis respectively. Macaro et al (2020) analysed quantitative and qualitative data derived from five EMI course teachers and 165 Beijing university students attending EMI courses whereas the case study by Tsang and Lo (2020) involved 14 Hong Kong primary school children for qualitative data analysis. The last of the discourse analysis study by Im (2020) gathered and examined data qualitatively based on Korean speakers of English from TV variety shows.

In essence, latest translanguaging ESL pedagogy research were predominantly participated by teachers, pre-service teachers, postgraduate and undergraduate students in 12 out of the 14 empirical studies found. This may be due to the fact that participants who are working or learning adults have higher literacy skills for researchers to collect clear and accurate information for research validity and reliability (Neuman, 2014) about translanguaging practices. With this said, further research could be done in favour of translanguaging ESL pedagogy for young ESL learners who are attending secondary, primary schools and preschools to provide richer data as Kleyn and García (2019) asserted translanguaging strategies in the ESL classroom are suitable and effective for emergent multilingual or bilingual students.

Moreover, referring to Appendix A and Table 2, six research articles assessed were of mixed-method research designs while another six articles adopted qualitative research designs for their studies followed by two articles using quantitative research designs. From this, it can also be concluded that there is currently some considerable research information representing views of educators and learners about translanguaging in the teaching and learning of ESL in smaller or larger research participant groups by the three types of research methodology or designs across seven articles.

\section{RQ 4: What were the multilingual backgrounds of the participants involved?}

The multilingual and bilingual backgrounds of the research participants found in 14 empirical research articles were diverse with relation to researching about translanguaging in the ESL classroom. All participants found in the research know and spoke the English language as a prerequisite. Three empirical research by Eisenstein Ebsworth et al (2018); Lindahl (2020); Lindahl et al (2020) had bilingual teachers or preservice teachers who spoke Spanish as L1 and English as L2. Additionally, Lindahl (2020) included teachers who speak French and English working in an American public school district in her research as well. In Qiu's and Fang's (2019) and Macaro's et al. (2020) study, most of their research participants comprised of native Chinese speakers learning ESL. Fourthly, bilingual Arabic and English college students were the focus in the translanguaging research by Akbar and Taqi (2020) and Holi Ali (2020). Im's (2020) empirical study revolved around research subjects who speak Korean natively and English as their L2 from the media in Korea. Next, Hong Kong primary school children speaking native Cantonese and learning ESL were the participants in the research by Tsang and Lo (2020). On the other hand, Turnbull (2017) investigated translingualism in the ESL classroom among New Zealand bilingual undergraduates whose mother tongues were Korean, Chinese, Vietnamese. Schreibers' (2019) research chose multilingual Sri Lankan postgraduates and bilingual New York undergraduates to participate in his or her research. In facts, the Sri Lankan ESL postgraduates were fluent in the official languages of their home country, Sinhala and Tamil whereas the New York undergraduate consisted of native speakers of Chinese Spanish, Russian, Arabic, Gujarati, Burmese, Serbo-Croatian, Tagalog, Haitian Creole, African American Vernacular English and dialects of New York English. Comparatively, 400 university ESL 
learners that took part in Amin et al. (2020) study were multilinguals knowing at least Urdu and English with additional languages regional to Pakistan such as Pashto, Punjabi, Balochi or Sindhi. Last but not least, Malaysian multilingual or bilingual ESL teachers participated in studies by Wong and Yoong (2019) along with Tan and Low (2017). Some teachers could speak Chinese as their first language, Malay as the official language of Malaysia and English in the classroom while others could speak Malay and English.

The language backgrounds of all the research participants in the 14 empirical literature of recent translanguaging ESL pedagogy research indicated most participants were bilinguals followed by a small number of multilinguals. It was also proven that all authors of the reviewed articles thoroughly examined and acknowledged all languages used by their participants including global Englishes (Rose et al., 2021) for the cause of minimizing language elitism and promoting heteroglossic views of language use (Bailey, 2007; García, 2009; Goodman \& Tastanbek, 2021).

\section{RQ 5: What were the considerations regarding ESL translanguaging pedagogy found in the studies?}

Considerations regarding ESL translanguaging pedagogy were identified in all of the articles for this systematic literature review. First, when investigating Malaysian Chinese Independent Secondary School teachers' perspective of translanguaging (Wong \& Yoong, 2019), the practice can facilitate learning of English grammar and lexical items. Teachers could assess students' learning by allowing them to explain content taught in L1 and vice versa. Holi Ali (2020) also explained translanguaging with L1 functions as cognitive strategies for summarising, clarification, emphasis and reiteration of crucial academic contents.

Secondly, Wong and Yoong (2019) advised teachers who use translanguaging can better express their opinions, thoughts, ideas and feelings when addressing learners' behaviour then build positive rapport, improve language learning proficiency and interest with them as a result, especially among learners' with lower motivation and beginner English learners. In like manner, Holi Ali (2020) and Tan and Low (2017) agreed translanguaging in the ESL classroom increases classroom management efficiency, establish good relationships and reduce disciplinary issues.

The Malaysian study (Wong \& Yoong, 2019) pointed out the use of English language in the classroom with adolescent or secondary school ESL beginner learners continuously in long periods may cause said learners to have learning anxiety and perceive ESL learning negatively due to classroom expectations of needing learners to use English as constantly and accurately. Classrooms that use English exclusively may create a greater language barrier between teachers and students (Qiu \& Fang, 2019). To avoid such situations, intermittent practice of translanguaging by ESL teachers could alleviate learners' anxiety and encourage English language learning as they gradually feel confident or comfortable in using L2 skills while alternating between L1 and L2 in the language classroom (Akbar \& Taqi, 2020).

According to Eisenstein Ebsworth et al. (2018), pedagogical considerations for ESL translanguaging given from their research among English teachers in Puerto Rico suggested learners should be allowed to tap into all their linguistic resources in to relate and make sense of the English language content being learnt. The ability to speak and write in two languages referred to as bilingualism and biliteracy or additive bilingualism where students' L1 are developed along with L2 should be supported by translanguaging teachers in the ESL classroom (Ebsworth et al., 2018; Tan \& Low, 2017). When teachers strive to develop 
students' native language and ESL with translingualism, learners will be able to enrich their professional and personal development with recognition of their cultural background while providing opportunities of learning ESL meaningfully by connecting to their native language or culture. As a matter of fact, teachers practicing translanguaging pedagogy was proven by Tsang and Lo (2020) to improve learners' L1 and L2 skills simultaneously although at different progression rates through their practice of comparing aspects of both languages, explicit teaching of transferable skills between $L 1$ and $L 2$ then repetition of the skills as consolidation in a pluri-centric approach bilingual programme. ESL teachers should reassure learners to appreciate languages used as they actively discover similarities and differences between their L1 and L2 when translanguaging for students' L1 and L2 skills can be improved altogether too (Holi Ali, 2020). As a result, translingualism as an ESL pedagogy cultivates self-efficacy and self-directed language learning (Tsang \& Lo, 2020) that could lead learners to academic and professional success.

When planning and conducting translanguaging ESL pedagogy, Qiu and Fang (2019) proposed the incorporation of multimodal devices and L1 that stimulate learners' visual, auditory or kinaesthetic senses simultaneously to improve teaching and learning efficiency in the ESL classroom. Said authors suggested educators should know learners' L1 and be able to communicate with their native language so teachers can develop their intracultural and intercultural competencies for effective classroom management and teaching. There was an emphasis in the reviewed article that teachers who are native speakers of learners' L1 should be preferred when assigning to teach a group of ESL learners. The rationale presented was that local English teachers are possibly more familiar with the learners' learning habits and thinking styles whose cultural background are similar to them. These type of teachers could provide learners with purposeful English content knowledge in which students can apply in domestic and international related interests. By the same token, Tan and Low (2017) added that teachers and learners who share the similar language repertoire and cultures can develop better interpersonal relationships amongst themselves through showing higher levels of cultural appreciation or cultivation of learners. Nonetheless, ethnicity, gender and teaching experiences or qualifications of teachers may be sociocultural factors affecting their efficiency of translanguaging between learners' L1 and L2 in the ESL classroom (Tan \& Low, 2017). Teachers' should acquire sufficient knowledge and practice of translanguaging to ensure the benefits of the pedagogy in practice.

Amin et al (2020) acknowledged with the aforementioned authors on the heavy emphasis of English-only education could lead to the diminishing of learners' L1 and other regional exclusive languages. Therefore, translanguaging ESL pedagogy should be considered and accommodated by teachers so both English as a target language and learners' first languages become valuable assets for effective learning. For translanguaging ESL pedagogy to succeed, Amin et al (2020); Akbar and Taqi (2020) recommended flexible language policies as a solution so education systems can eliminate linguistic disparities and allow relevant authorities to provide for learners' linguistic needs and preferences.

With reference to Tan and Low's (2017) research about younger learners, ESL teachers providing their services to rural areas or ESL primary school classrooms with lower English proficiency learner should consider employing translanguaging strategies. Akbar and Taqi (2020) further complemented ESL translingualism may be more effective to improve language skills of learners with beginner to intermediate levels of English in their research with tertiary education students. Translanguaging pedagogy is preferred to reduce cognitive loads of 
bilinguals or multilinguals by allowing them access to all their linguistic resources when understanding a new language or knowledge which was also in tandem with the findings from Akbar and Taqi (2020); Tsang and Lo (2020); Macaro et al (2020); Ali (2020). Furthermore, Macaro et al (2020) elaborated considerations for translingualism as an ELT for explaining conceptually complex language and academic content extending to tertiary education syllabus due to learners' better chances of activating their prior knowledge to relate new information meaningfully. Akbar and Taqi (2020) further supported this notion, stating teachers and learners who use translanguaging can develop higher order thinking skills.

In translanguaging ESL pedagogy, collaborative and cooperative learning among peers are needed to progress students' language learning. Scaffolding learning with peers who use translanguaging can enable learners to understand elements of the target language taught (García, 2009; Holi Ali, 2020). Student-student and student-teacher translingual interactions in the ESL classroom allow learners to mutually negotiate, encode or decode meaning by employing linguistic, cultural and contextual resources from their L1 and L2 (Im, 2020). Notably, Schreiber (2019) and Im (2020) emphasized that translingualism pedagogy should shift away from unrealistic ESL ideologies such as speaking with an English native-like accent with perfect language accuracy but consider the development of an individuals' English communication competence as the practical aim for ESL learners. In detail, translingualism provides opportunity for teachers to recognize and acknowledge various languages of the world including the localisations of Englishes in which teachers will need to consider translanguaging pedagogy developing learners' communications across diverse situations that may involve varieties of languages (Im, 2020). Thereupon, Lindahl (2020) and Lindahl et al (2020) asserted that educators need to steer away from strict standardisation of past ELT and consider translanguaging pedagogy so they could understand ways ESL learners can use or acquire the target language by using the Critical Multilingual Language Awareness (CMLA) from García (2009) framework as guidance. The CMLA framework calls for teachers to create a learning environment where learners' have linguistic tolerance and plurilingualism awareness when translanguaging with their full linguistic repertoire in the classroom as well as leverage the practice of minoritised languages and the target language to be equally significant. Therefore, ESL educators must be open-minded and confident towards learners' linguistic knowledge as a resource for successful learning (Akbar \& Taqi, 2020; Turnbull, 2017) to extend their teaching repertoire with translanguaging.

ESL teachers need to make careful and informed decisions when teaching with translanguaging since it is possible for learners to become confuse and cognitively overloaded which cause them to perceive the act as counterintuitive (Holi Ali, 2020; Tsang \& Lo, 2020; Turnbull, 2017). With this in mind, Turnbull (2017) urged educators to practice translanguaging with learners strategically using a set of rules for the threshold of L1 meshing so a balance is achieved. The rules set must be so that L1 usage does not reduce exposure to L2 or have learners over relying on L1 when participating in ESL learning activities. Teachers should also be well aware that translanguaging may occur lesser between advanced ESL proficiency learners who only resort to L1 use when facilitating for better flow of conversations, resolving miscommunications, engaging in advanced problem-solving or learning complex content (Macaro et al., 2020; Turnbull, 2017). Equally, translingualism may be needed more frequently among beginner ESL learners and gradually decrease as higher L2 proficiency learners gain diversified learning strategies (Turnbull, 2017). 
Overall, the considerations regarding ESL translanguaging pedagogy in the 14 empirical studies reviewed were found to be extensive for planning, implementation and research purposes on the subject matter. English language educators and researchers may refer the factors and issues discussed in this section about effective translingualism for ELT.

\section{Implications and Conclusion}

In this systematic literature review, 14 empirical published and peer-reviewed research articles were identified and analysed for recent information on translanguaging pedagogy in the ESL classroom within five years from 2017-2020 according to the research questions formulated for this research paper. Known research aims on translanguaging for ESL teaching and learning were mainly on learners' and teachers' views of the pedagogical practice followed by functions, effects and factors influencing translanguaging use of mentioned groups. Next, recent translanguaging research took place in several geographical settings with no location that was densely explored although all studies reviewed showed areas where ESL learners are found to be a requisite. It was also determined that latest translanguaging research trends concentrated on data collection from teachers, pre-service-teachers, postgraduates and undergraduates more regarding their views on ESL translanguaging with mixed method, qualitative and quantitative research methodology seconded by significantly lesser investigations on younger ESL learners as research participants and other research aims mentioned before. As for the fourth research question of this paper, translanguaging research in all the authors' written article acknowledged and brought attention to the variety of languages learners use as L1 when considering translanguaging practices in the ESL classroom so their cultural identity could be continually preserved and developed. The fifth research questions revealed in-depth considerations for translanguaging as a pedagogical practice in the ESL classroom which included teachers' sufficient knowledge of learners' L1 and cultural backgrounds as readiness for teaching ESL with translanguaging, incorporation of collaborative and cooperative modes of learning, suitability of the practice among learners of varying proficiency levels and pedagogical situations apt for the practice to take place as examples.

On a final note, this review provided new insights for ESL education practitioners, researchers, policy makers and other relevant parties on recent data regarding translanguaging as an ESL pedagogy or ELT. The findings discussed in each research question contributed knowledge regarding prevailing research objectives, sites, participants' backgrounds and pedagogical considerations related to translanguaging in the ESL classroom. More in-depth studies could be done on the lesser researched areas of the subject matter discussed above or concepts that were not mentioned in this paper to bridge the gaps and advance the research on translingualism for ELT. ESL educators and researchers can consult this systematic review as a guidance when considering optimal and practical ways to conduct translingualism for teaching ESL. For instance, translingualism as an ESL pedagogy research may broaden its scope in terms of investigating ESL learners who speak multiple languages as a comparison to data obtained from ESL bilingual learners regarding the effects, views, influences and functions of learning English through translanguaging. Next, Lesser quantitative, qualitative and mixed-method research on the functions of translanguaging, factors influencing participants' view of translanguaging and effects of translanguaging pedagogy on ESL learners can be investigated by relevant parties for further development of the concept in academia. Additional research on translanguaging pedagogy could expand on 
the functions of translanguaging in ESL teaching and learning, the impact of translanguaging as an ESL pedagogy on students' academic achievements and the influences affecting teachers' and students' view of the translanguaging practice.

Regardless, this study has some limitations to consider as the results from literature synthesis may simplify or overlook some points of interests offered in the empirical studies. The empirical studies found were limited to one database and pertaining to a set string of keywords searched in Google Scholar. However, the quality of the empirical studies was of reliable sources by adhering to the systematic literature search process proposed.

\section{Corresponding Author}

Azlina Abdul Aziz is a TESL lecturer at the Faculty of Education, UKM. She has an Ed.D. in Teaching of English from Teachers College, Columbia University, U.S.A. Her research interests are in the Teaching and Learning of Literature and Teacher Education in TESL. She is interested in how literary texts and personal narrative may be utilised to help students to examine the social, cultural and political issues in a particular context.

\section{References}

Akbar, R. S. S., \& Taqi, H. A. (2020). Translanguaging as an ESL Learning strategy: A case study in Kuwait. International Journal of Higher Education, 9(6), 54. https://doi.org/10.5430/ijhe.v9n6p54

Amin, R. U., Ahmed, F., \& Haidar, S. (2020). Linguistic divide and the fate of regional language(s) in Pakistani education systems: Edvidence from graduate students. Academic Journal of Social Sciences, 4(2), 231-250.

Bailey, B. (2007). Heteroglossia and boundaries. In M. Heller (Ed.), Bilingualism: A social approach (pp. 257-274). Palgrave Macmillan.

Beare, K. (2020). How many people learn English? https://www.thoughtco.com/how-manypeople-learn-english-globally-1210367

Briner, R. B., \& Denyer, D. (2012). Systematic review and evidence synthesis as a practice and scholarship tool. In D. M. Rousseau (Ed.), The Oxford Handbook of Evidence-based Management (pp. 112-129). Oxford University Press.

Conteh, J. (2018). Translanguaging. ELT Journal, 72(4), 445-447. https://doi.org/10.1093/elt/ccy034

Creese, A., \& Blackledge, A. (2010). Translanguaging in the bilingual classroom: A pedagogy for learning and teaching. The Modern Language Journal, 94, 103-115.

Del Valle, J. (2000). Monoglossic policies for a heteroglossic culture: Misinterpreted multilingualism in modern Galicia. Language and Communication, 20, 105-132.

Deroo, M. R., \& Ponzio, C. (2019). Confronting ideologies: A discourse analysis of in- service teachers ' translanguaging stance through an ecological lens translanguaging stance through an ecological lens. Bilingual Research Journal, 1-18.

https://doi.org/10.1080/15235882.2019.1589604

Eisenstein Ebsworth, M., Ebsworth, T. J., \& Cai, C. (2018). English acquisition in Puerto Rico: Teachers' insights. Bilingual Research Journal, 41(1), 69-88.

https://doi.org/https://doi.org/10.1080/15235882.2017.1413441

Ferguson, C. A. (1959). Diglossia. Word, 15, 325-340.

García, O. (2009). Education, multilingualism and translanguaging in the 21st century. In A. K. 
Mohanty, M. Panday, R. Phillipson, \& T. Skutnabb-Kangas (Eds.), Multilingual Education for Social Justice: Globalising the Local (pp. 140-158). Orient Blackswan.

García, O., \& Hesson, S. (2015). Translanguaging frameworks for teachers: Macro and micro perspectives. In A. Yiakoumetti (Ed.), Multlingualism and Langauge in Education (pp. 221-241). Cambridge University Press.

Garcia, O., \& Wei, L. (2014). Translanguaging and education. In Translanguaging: Language, bilingualism, and education (pp. 63-77). Palgrave Pivot, London.

Gardner-Chloros, P. (2009). Code-switching. Cambridge University Press.

Goodman, B., \& Tastanbek, S. (2021). Making the shift from a codeswitching to a translanguaging lens in English language teacher education. TESOL Quarterly, 55(1), 2953. https://doi.org/10.1002/tesq.571

Hart, C. (2018). Doing a literature review: releasing the research imagination (2nd ed.). SAGE Publications.

Ali, H. I. (2020). Omani Students' Coping Strategies in an English Medium Engineering Programme. Arab World English Journal, 11(2), 361-382. https://doi.org/https://dx.doi.org/10.24093/awej/vol11no2.25

Hopewell, S., Clarke, M., \& Mallett, S. (2005). Grey literature and systematic reviews. In H. R. Rothstein, A. J. Sutton, \& M. Borenstein (Eds.), Publication bias in meta-analysis: Prevention, assessment and adjustments (pp. 48-72). John Wiley \& Sons.

$\mathrm{Hu}$, J. (2020). Translanguaging in ESL / EFL Classes : A Review Paper. Journal of Contemporary Educational Research, 4(9).

Im, J. (2020). Introduction to Translingualism: Some teaching implications for Korean teachers of English. Korean Journal of Applied Linguistics, 36(1), 33-63. https://doi.org/http://dx.doi.org/10.17154/kjal.2020.3.36.1.33

Kamwangamalu, N. M. (2010). Multilingualism and codeswitching in education. In N. H. Hornberger \& S. L. McKay (Eds.), Sociolinguistics and language education (pp. 116-142). Springer.

Khan, K. S., Kunz, R., Kleijnen, J., \& Antes, G. (2003). Five steps to conducting a systematic review. Journal of the Royal Society of Medicine, 96(3), 118-121.

Kleyn, T., \& García, O. (2019). Translanguaging as an act of transformation: restructuring teaching and learning for emergent bilingual students. In L. C. De Oliveira (Ed.), The Handbook of TESOL in K-12 (pp. 69-82). John Wiley \& Sons. https://doi.org/10.1002/9781119421702.ch6

Lewis, G., Jones, B., \& Baker, C. (2012). Translanguaging: origins and development from school to street and beyond. Educational Research and Evaluation: An International Journal on Theory and Practice, 18(7), 641-654.

Lindahl, K. (2020). Connecting Ideology and Awareness: Critical Multilingual Awareness in CLIL Contexts. English Teaching and Learning, 44, 211-228. https://doi.org/https://doi.org/10.1007/s42321-020-00053-3 ORIGINAL

Lindahl, K., Hansen-Thomas, H., Baecher, L., \& Stewart, M. A. (2020). Study abroad for critical multilingual language awareness development in ESL and bilingual teacher candidates. Tesl-Ej, 23(4), 1-13.

Lüpke, F. (2020). Standardization in highly multilingual contexts: The shifting interpretations, limited reach, and great symbolic power of ethnonationalist visions. In The Cambridge handbook of standard languages. Cambridge University Press.

Macaro, E., Tian, L., \& Chu, L. (2020). First and second language use in English medium 
DEVELOPMENT

Vol. 10, No. 3, 2021, E-ISSN: 2226-6348 @ 2021 HRMARS

instruction contexts. Language Teaching Research, 24(3), 382-402. https://doi.org/10.1177/1362168818783231

MacSwan, J. (2017). A multilingual perspective on translanguaging. American Educational Research Journal, 54(1), 167-201.

Matamoros-González, J. A., Asunción Rojas, M. a., Pizarro Romero, J., Vera-Quiñonez, S., \& Soto, S. T. (2017). English language teaching approaches: a comparison of the Grammartranslation, Audiolingual, Communicative, and Natural Approaches. Theory and Practice in Language Studies, 7(11). https://doi.org/10.17507/tpls.0711.04

Moher, D., Liberati, A., Tetzlaff, J., Altman, D., \& The PRISMA Group. (2009). Preferred Reporting Items for Systematic Reviews and Meta-Analyses: The PRISMA Statement. PLOS Medicine, 6(7), e1000097.

https://doi.org/https://doi.org/10.1371/journal.pmed.1000097

Myers-Scotton, C. (1993). Common and uncommon ground: Social and structural factors in codeswitching. Language in Society, 22, 475-503.

Neuman, W. L. (2014). Social research methods: qualitative and quantitative approaches (7th ed.). Pearson Education Limited.

Qiu, X., \& Fang, C. (2019). Creating an effective English-Medium Instruction (EMI) classroom: Chinese undergraduate students' perceptions of native and non-native English-speaking content teachers and their experiences. International Journal of Bilingual Education and Bilingualism, 1-15. https://doi.org/https://doi.org/10.1080/13670050.2019.1707769

Rose, H., Mckinley, J., \& Galloway, N. (2021). Global Englishes and language teaching: A review of pedagogical research. Language Teaching, 54, 157-189.

https://doi.org/10.1017/S0261444820000518

Schreiber, B. R. (2019). "More Like You": Disrupting native speakerism through a multimodal online intercultural exchange. TESOL Quarterly, 53(4), 1115-1138. https://doi.org/https://doi.org/10.1002/tesq.534

Tan, N. S., \& Low, H. M. (2017). Socio-environmental factors impacting on teachers' attitudes towards code-switching in ESL classrooms in a rural district in Malaysia. Kajian Malaysia, 35(2), 105-125. https://doi.org/https://doi.org/10.21315/km2017.35.2.6

Ting, S., \& Jintang, L. (2020). Teacher and students' translanguaging practices in a Malaysian preschool Teacher and students ' translanguaging practices in a. International Journal of Early Years Education, 1-15. https://doi.org/10.1080/09669760.2020.1850429

Tsang, A., \& Lo, F. (2020). Bilingual education through a pluri-centric approach: A case study of the effects of simultaneously learning two languages on $L 1$ and $L 2$ reading and writing proficiency. Studies in Educational Evaluation, 67, 1-10. https://doi.org/https://doi.org/10.1016/j.stueduc.2020.100927

Turnbull, B. (2017). Examining pre-service ESL teacher beliefs: Perspectives on first language use in the second language classroom. Journal of Second Language Teaching and Research, 6, 1-27.

Uman, L. S. (2011). Systematic reviews and meta-analyses. Journal of the Canadian Academy of Child and Adolescent Psychiatry, 20(1), 57-59.

Williams, C. (1994). Arfarniad o ddulliau dysgu ac addysgu yng nghyd-destun addysg uwchradd ddwyieithog [Evaluation of teaching and learning methods in the context of bilingual secondary education]. University of Wales.

Williams, C. (2000). Bilingual teaching and language distribution at 16+. International Journal of Bilingual Education and Bilingualism, 3(2), 129-148. 
INTERNATIONAL JOURNAL OF ACADEMIC RESEARCH IN PROGRESSIVE EDUCATION AND DEVELOPMENT

Vol. 10, No. 3, 2021, E-ISSN: 2226-6348 @ 2021 HRMARS

Wong, Y., Von, \& Yoong, D. (2019). ESL teachers' perceptions and practices of code-switching in a Malaysian Chinese independent secondary school. Journal of Modern Languages, 29, 45-66. https://doi.org/https://doi.org/10.22452/jml.vol29no1.3 